\title{
Parameter and State Estimation of Shear Buildings Using Spline Interpolation and Linear Integral Filters
}

\author{
Antonio Concha $\mathbb{( D D}^{1}$ and Luis Alvarez-Icaza $\left.{ }^{\mathbb{D}}\right)^{2}$ \\ ${ }^{1}$ Facultad de Ingeniería Mecánica y Eléctrica, Universidad de Colima, 28400 Coquimatlán, COL, Mexico \\ ${ }^{2}$ Instituto de Ingeniería, Universidad Nacional Autónoma de México, 04510 Coyoacán, CDMX, Mexico \\ Correspondence should be addressed to Luis Alvarez-Icaza; alvar@pumas.iingen.unam.mx
}

Received 25 February 2018; Accepted 18 April 2018; Published 19 July 2018

Academic Editor: Daniele Baraldi

Copyright (c) 2018 Antonio Concha and Luis Alvarez-Icaza. This is an open access article distributed under the Creative Commons Attribution License, which permits unrestricted use, distribution, and reproduction in any medium, provided the original work is properly cited.

\begin{abstract}
A parameter identification method and a high gain observer are proposed in order to identify the model and to recover the state of a seismically excited shear building using acceleration responses of the ground and instrumented floors levels, as well as the responses at noninstrumented floors, which are reconstructed by means of cubic spline shape functions. The identification method can be implemented online or offline and uses Linear Integral Filters, whose bandwidth must enclose the spectrum of a seismically excited building. On the other hand, the proposed state observer estimates the displacements and velocities of all the structure floors using the model estimated by the identification method. The observer allows obtaining a fast response and reducing the state estimation error, while depending on a single gain. The performance of the parameter and state estimators is verified through experiments carried out on a five-story small scale building.
\end{abstract}

\section{Introduction}

Acceleration responses of the building floor, obtained when it is excited through earthquakes or wind, are employed for parameter and state estimation of the structure or for monitoring its health. Because of cost, most of the buildings are instrumented only at a few stories. For this reason, the estimation and health monitoring of a structure must be carried out using as data only the responses of the instrumented stories.

Parameter and state estimation of civil structures using recorded responses has attracted the attention of a large number of researchers around the world during the last four decades. Some relevant references for not fully instrumented structures [1-15] are revised here. Amini and Hedayati [1] propose a Sparse Component Analysis approach for modal identification of structures, where the number of sensors is smaller than the number of active modes. References [2-5] employ the extended Kalman filter in order to estimate the parameters of a structure, as well as its displacements and velocities at noninstrumented floors. This filter is obtained by linearizing a nonlinear state equation that considers the building parameters as states. Nevertheless, some poles of the linearized model may lie on the imaginary axis and, as a consequence, some parameter and/or states may be unbounded. On the other hand, Zhou et al. [6] recursively estimate the stiffness and damping ratios of a structure using the first two derivatives of Log-Likelihood Measure and the knowledge of the building mass. Mukhopadhyay et al. [7] develop mode shape normalization and expansion approaches that utilize the topology of the structural matrices for estimating the mass and stiffness parameters of a building under base excitation; authors obtain the global identifiability requirements for their methodology and show that, for estimating the structural parameters, the number $P$ of instrumented floors should satisfy $P \geq \sqrt{n}$ and $P \geq(1+$ $\sqrt{4 n-3}) / 2$ if the floor masses at sensor locations are known and if the total mass of the structure is known, respectively, where $n$ is the number of floors of the building. Yuan et al. [8] estimate the mass and stiffness matrices of shear buildings using a method depending on the first two orders of modal data. References [9-11] present OKID identification 
techniques, which determine the Markov parameters of a Kalman observer in order to identity a building model. These techniques identify a discrete time model of the structure, but if the sampling frequency of responses is too high in relation to the dominate frequencies of the structure, then the poles of the estimated model lie close to the unit circle in the complex domain, which may produce parameter estimates statistically ill-defined [16]. Kaya el al. [12] identify a structure using the Transfer Matrix formulation of the response, where the histories at noninstrumented floors are offline reconstructed using the Mode Shape Based Estimation (MSBE) method, which assume that modal shapes can be approximated as a linear combination of the mode shapes of a shear beam and a bending beam. It is worth mentioning that using MSBE requires solving two partial differential equations and the knowledge of the modal acceleration of the instrumented floors. Hegde and Sinha [13] estimate the modal parameters of a seismically excited, torsionally coupled building using limited number of responses; modal parameters are extracted using an offline estimation methodology based on the principles of the Natural Excitation Technique and the Eigen Realization Algorithm; authors use a cubic shape function for estimating the responses at noninstrumented floors. On the other hand, $[14,15]$ carried out structural identification using ambient vibration measurements and offline algorithms. Huang [14] proposes a procedure for identifying the dynamics characteristics of a shear building using the multivariate ARV model, whereas Chakraverty [15] estimates mass and stiffness parameters from modal test data and the Holzer criteria.

This manuscript presents (1) an identification technique to identify the parameters of the model of a seismically excited shear building using a limited number of responses and (2) a high gain state observer that employs the identified parameter in order to estimate the displacements and velocities of both instrumented and noninstrumented floors. The parameter and state estimators rely on the acceleration measurements of ground and some instrumented floors. The acceleration at noninstrumented floors is reconstructed by means of cubic shape spline functions that use the available measurements. The state observer gain is easily designed and depends on a positive parameter that guarantees the stability and a fast response of the observer. On the other hand, the parameter estimator uses a parameterization with the following features: (1) assuming that building has Rayleigh damping; (2) containing a vector whose entries are the stiffness/mass ratios of the building, which are estimated through a Least Squares algorithm; and (3) employing Linear Integral Filters (LIF) that attenuate low and high frequency noise of the measured and reconstructed responses. The LIF were previously employed in Garrido and Concha [17] for parameter estimation of fully instrumented structures. In this manuscript, the application of these filters is extended to estimate the model of buildings instrumented at only few floor levels. Moreover, by considering Rayleigh damping for the structure, the number of parameters of the proposed parameterization is the half of the one corresponding to the parameterization in [17]. In comparison with techniques in [7-15] described above, the proposed parameter estimator allows attenuating low and high frequency noise of both measured and reconstructed acceleration and can be implemented online at a low computational effort.

It is worth mentioning that the cubic spline functions, employed by the proposed parameter and state estimators, are designed following the ideas in $[18,19]$. Moreover, the design of these functions does not require the knowledge of the modal shapes of the structure, and they allow recursive estimation of the responses at noninstrumented floors. Techniques in [20-26] are also able to estimate the unmeasured floor level responses, but unlike the cubic spline functions they are implemented offline and require an estimated building model, which may be unavailable.

The paper has the following structure. Sections 2 and 3 present the shear building model and its parameterization, respectively. The cubic spline functions used for estimating the floor responses at noninstrumented floors are described in Section 4. Section 5 shows the methodology for estimating the building parameters. The proposed high gain observer is described in Section 6. Experiment results using the parameter and state estimators are shown in Section 7. Finally, Section 8 includes the conclusions of this paper.

\section{Mathematical Model of a Shear Building}

The dynamics of a shear building with $n$ stories subjected to earthquake or base motion is described through the following mathematical model [27]:

$$
\begin{aligned}
& \mathbf{M}\left(\ddot{\mathbf{x}}(t)+\mathbf{l} \ddot{x}_{g}(t)\right)+\mathbf{C} \dot{\mathbf{x}}(t)+\mathbf{K} \mathbf{x}(t)=\mathbf{0} \\
& \mathbf{x}(t)=\left[x_{1}(t), \ldots, x_{n}(t)\right]^{T}, \\
& \dot{\mathbf{x}}(t)=\left[\dot{x}_{1}(t), \ldots, \dot{x}_{n}(t)\right]^{T} \\
& \ddot{\mathbf{x}}(t)=\left[\ddot{x}_{1}(t), \ldots, \ddot{x}_{n}(t)\right]^{T}, \\
& \mathbf{l}=[1,1, \ldots, 1]^{T} \\
& \ddot{\mathbf{x}}_{\mathbf{a}}(t)=\ddot{\mathbf{x}}(t)+\mathbf{l} \ddot{x}_{g}(t)=\left[\ddot{x}_{a_{1}}(t), \ldots, \ddot{x}_{a_{n}}(t)\right]^{T},
\end{aligned}
$$

where variables $x_{i}(t), \dot{x}_{i}(t)$, and $\ddot{x}_{i}(t)$ are, respectively, the displacement, velocity, and acceleration of the $i$ th floor, which are measured with respect to the basement. Signal $\ddot{x}_{a_{i}}(t)$ represents the absolute acceleration at the $i$ th floor, and $\ddot{x}_{g}(t)$ is the ground acceleration produced by earthquake. Moreover, $\mathbf{M}$ and $\mathbf{K}$ are the mass and stiffness matrices, respectively, which are defined as

$$
\begin{aligned}
\mathbf{M} & =\left[\begin{array}{cccc}
m_{1} & 0 & \cdots & 0 \\
0 & m_{2} & \cdots & 0 \\
\vdots & \vdots & \ddots & \vdots \\
0 & 0 & \cdots & m_{n}
\end{array}\right], \\
\mathbf{K} & =\left[\begin{array}{cccc}
k_{1}+k_{2} & -k_{2} & \cdots & 0 \\
-k_{2} & k_{2}+k_{3} & \cdots & 0 \\
\vdots & \vdots & \ddots & \vdots \\
0 & 0 & \cdots & k_{n}
\end{array}\right]
\end{aligned}
$$


The entry $k_{i}(i=1,2, \ldots, n)$ is the column lateral stiffness between the $i$ th and $(i-1)$ th floors.

The building has Rayleigh damping that is represented through the following matrix $\mathbf{C}$ :

$$
\mathbf{C}=a_{0} \mathbf{M}+a_{1} \mathbf{K}
$$

Parameters $a_{0}$ and $a_{1}$ are constant, and they are computed as [27]

$$
\frac{1}{2}\left[\begin{array}{cc}
\frac{1}{\omega_{i}} & \omega_{i} \\
\frac{1}{\omega_{j}} & \omega_{j}
\end{array}\right]\left[\begin{array}{l}
a_{0} \\
a_{1}
\end{array}\right]=\left[\begin{array}{l}
\zeta_{i} \\
\zeta_{j}
\end{array}\right],
$$

where $\zeta_{v}$ and $\omega_{v}, v=i, j$, are the damping ratio and natural frequency of the $v$ th structural mode, respectively.

Remark 1. Computing the constants $a_{0}$ and $a_{1}$ in (6) requires knowledge of the parameters $\omega_{i}, \omega_{j}, \zeta_{i}$, and $\zeta_{j}$ that corresponds to the $i$ th and $j$ th structural modes. Natural frequencies $\omega_{i}$ and $\omega_{j}$ can be extracted by means of the Fourier spectra of the building responses produced by ambient or force excitations. On the other hand, the parameters $\zeta_{i}$ and $\zeta_{j}$ can be obtained with any of the following techniques:

(i) Half-power bandwidth method [28], which estimates the damping ratios using the peaks of the response Fourier spectra

(ii) Logarithmic Decrement method, which computes the damping ratios in the time domain by means of the ratio between the amplitudes of any two closely adjacent peaks of acceleration responses [29]

(iii) Enhanced Frequency Domain Decomposition method that estimates the modal damping from the Singular Value plots of recorded acceleration [30]

(iv) Using the recommended damping values shown in Table 11.2.1 from [27], which depend on the type and condition of the structure.

The state space model corresponding to the differential equation (1) is given by

$$
\begin{aligned}
& \dot{\boldsymbol{\eta}}(t)=\mathbf{A} \boldsymbol{\eta}(t)+\mathbf{B} \ddot{x}_{g}(t) \\
& \mathbf{A}=\left[\begin{array}{cc}
\mathbf{O}_{\mathbf{n} \times \mathbf{n}} & \mathbf{I}_{\mathbf{n} \times \mathbf{n}} \\
-\mathbf{M}^{-1} \mathbf{K} & -\mathbf{M}^{-1} \mathbf{C}
\end{array}\right], \\
& \mathbf{B}=\left[\begin{array}{c}
\mathbf{O}_{\mathbf{n} \times \mathbf{1}} \\
-\mathbf{l}
\end{array}\right] \\
& \begin{aligned}
\boldsymbol{\eta}(t) & =\left[\mathbf{x}^{\mathbf{T}}(t), \dot{\mathbf{x}}^{\mathbf{T}}(t)\right]^{T} \\
& =\left[x_{1}(t), x_{2}(t), \ldots, x_{n}(t), \dot{x}_{1}(t), \dot{x}_{2}(t), \ldots, \dot{x}_{n}(t)\right]^{T},
\end{aligned}
\end{aligned}
$$

where $\mathbf{I}_{\mathbf{p} \times \mathbf{r}}$ and $\mathbf{O}_{\mathbf{p} \times \mathbf{r}}$ are the identity and zero matrices with size $p \times r$, respectively.

The output equation of the state space model depends on the absolute acceleration of the instrumented floors. Let $P$ be the number of instrumented floors, and let $i=1,2, \ldots, P$ be the $i$ th instrumented floor numbered from bottom to top of the building. Then, the output equation is given by

$$
\begin{aligned}
\mathbf{y}(t) & =\Gamma \ddot{\mathbf{x}}_{\mathbf{a}}(t)=\Gamma\left[\ddot{\mathbf{x}}(t)+\mathbf{l} \ddot{x}_{g}(t)\right]=\Gamma \mathbf{D} \boldsymbol{\eta}(t), \\
\mathbf{D} & =\left[-\mathbf{M}^{-1} \mathbf{K},-\mathbf{M}^{-1} \mathbf{C}\right],
\end{aligned}
$$

where $\Gamma \in R^{P x n}$ is the localization matrix of the accelerometers, whose entries are defined as

$$
\begin{aligned}
& \Gamma_{i, j} \\
& = \begin{cases}1 & \text { if the } j \text { th floor is the } i \text { th instrumented story } \\
0 & \text { otherwise }\end{cases}
\end{aligned}
$$

\section{Model Parameterization}

Assume first that all the stories are instrumented; it means that $\Gamma=\mathbf{I}_{n x n}$; then substituting (5) into (1) yields

$$
\ddot{\mathbf{x}}(t)+\mathbf{l} \ddot{x}_{g}(t)=-\mathbf{M}^{-1}\left[a_{0} \mathbf{M}+a_{1} \mathbf{K}\right] \dot{\mathbf{x}}(t)-\mathbf{M}^{-1} \mathbf{K} \mathbf{x}(t)
$$

Equation (12) can be rewritten as

$$
\ddot{\mathbf{x}}_{\mathbf{a}}(t)+a_{0} \dot{\mathbf{x}}(t)=-a_{1} \mathbf{M}^{-1} \mathbf{K} \dot{\mathbf{x}}(t)-\mathbf{M}^{-\mathbf{1}} \mathbf{K} \mathbf{x}(t)
$$

Define the following equalities:

$$
\begin{aligned}
& \mathbf{M}^{-\mathbf{1}} \mathbf{K} \dot{\mathbf{x}}(t)=\dot{\mathbf{u}}(t) \boldsymbol{\theta}, \\
& \mathbf{M}^{-\mathbf{1}} \mathbf{K} \mathbf{x}(t)=\mathbf{u}(t) \boldsymbol{\theta},
\end{aligned}
$$

where $\dot{\mathbf{u}}(t)$ is the time derivative of $\mathbf{u}(t)$ and

$$
\begin{aligned}
& \boldsymbol{\theta}=\left[\begin{array}{lllll}
\frac{k_{1}}{m_{1}} & \frac{k_{2}}{m_{1}} & \frac{k_{2}}{m_{2}} & \cdots & \frac{k_{n}}{m_{n}}
\end{array}\right]^{T}=\left[\begin{array}{lllll}
\theta_{1} & \theta_{2} & \theta_{3} & \cdots & \theta_{2 n-1}
\end{array}\right]^{T} \\
& \mathbf{u}(t) \\
& =-\left[\begin{array}{ccccc}
x_{1}(t) & x_{1}(t)-x_{2}(t) & 0 & \cdots & 0 \\
0 & 0 & x_{2}(t)-x_{1}(t) & \cdots & 0 \\
\vdots & \vdots & \vdots & \vdots & \vdots \\
0 & 0 & 0 & \cdots & x_{n}(t)-x_{n-1}(t)
\end{array}\right]
\end{aligned}
$$

Using equalities in (14) leads to the following expression:

$$
\ddot{\mathbf{x}}_{\mathbf{a}}(t)+a_{0} \dot{\mathbf{x}}(t)=\left[a_{1} \dot{\mathbf{u}}(t)+\mathbf{u}(t)\right] \boldsymbol{\theta}
$$

In order to use only acceleration measurements and to attenuate low and high frequency measurement noise, (16) is first derived three times with respect to time, and, subsequently, the resulting expression is integrated five times over finite time intervals, thus obtaining

$$
I_{5}\left\{\ddot{\mathbf{x}}_{\mathbf{a}}^{(3)}(t)+a_{0} \ddot{\mathbf{x}}^{(2)}(t)\right\}=I_{5}\left\{a_{1} \ddot{\mathbf{u}}^{(2)}(t)+\ddot{\mathbf{u}}^{(1)}(t)\right\} \boldsymbol{\theta}
$$

The superindex (i) denotes the $i$ th time derivative of the corresponding signal; and $I_{5}\{\cdot\}$ is a Linear Integral Filter (LIF). The general definition a LIF is given by

$$
\begin{aligned}
I_{\alpha} & \{\rho(t)\} \\
& =\frac{1}{\delta^{\alpha-1}} \int_{t-\delta}^{t} \int_{\tau_{1}-\delta}^{\tau_{1}} \cdots \int_{\tau_{\alpha-1}-\delta}^{\tau_{\alpha-1}} \rho\left(\tau_{\alpha}\right) d \tau_{\alpha} \cdots d \tau_{1},
\end{aligned}
$$


where $\alpha$ is the number of integrations, and $\delta$ is the time integration window length defined as

$$
\delta=\hbar T_{s},
$$

where $\hbar>0$, and $T_{s}$ is the sampling period of $\rho$.

The Laplace transform of $I_{\alpha}\{\rho(t)\}$ in (18) is given by [17]

$$
\begin{aligned}
\mathscr{L}\left[I_{\alpha}\{\rho(t)\}\right] & =\delta G_{\alpha}(s) \mathscr{L}[\rho(t)] \\
G_{\alpha}(s) & =\left(\frac{1-e^{-\delta s}}{\delta s}\right)^{\alpha}, \\
\left|G_{\alpha}(j \omega)\right| & =\left|\frac{\sin \left(\pi \omega / \omega_{c}\right)}{\left(\pi \omega / \omega_{c}\right)}\right|^{\alpha}, \\
\omega_{c} & =\frac{2 \pi}{\delta} \\
f_{c} & =\frac{1}{\delta}
\end{aligned}
$$

Terms $\omega_{c}$ and $f_{c}$ determine the bandwidth of the $\alpha$ th-order low-pass filter $G_{\alpha}(s)$ in $\mathrm{rad} / \mathrm{s}$ and $\mathrm{Hz}$, respectively.

Equation (17) is equivalent to the following parameterization, which will be employed for parameter identification purposes:

$$
\begin{aligned}
\chi(t)= & \phi(t) \boldsymbol{\theta} \\
\chi(t)= & \frac{1}{\delta^{3}} I_{2}\left\{\sum_{j=0}^{3}\left(\begin{array}{l}
3 \\
j
\end{array}\right)(-1)^{j} \ddot{\mathbf{x}}_{\mathbf{a}}(t-j \delta)\right\} \\
& +\frac{a_{0}}{\delta^{2}} I_{3}\left\{\sum_{j=0}^{2}\left(\begin{array}{l}
2 \\
j
\end{array}\right)(-1)^{j} \ddot{\mathbf{x}}(t-j \delta)\right\} \\
\phi(t)= & \frac{a_{1}}{\delta^{2}} I_{3}\left\{\sum_{j=0}^{2}\left(\begin{array}{l}
2 \\
j
\end{array}\right)(-1)^{j} \ddot{\mathbf{u}}(t-j \delta)\right\} \\
& +\frac{1}{\delta} I_{4}\left\{\sum_{j=0}^{1}\left(\begin{array}{l}
1 \\
j
\end{array}\right)(-1)^{j} \ddot{\mathbf{u}}(t-j \delta)\right\}
\end{aligned}
$$

It is important to mention that variables $\chi(t)$ and $\phi(t)$ can be written in the Laplace domain as

$$
\begin{aligned}
& \mathscr{L}[\chi(t)]=\mathscr{H}_{3}(s) \mathscr{L}\left[\ddot{\mathbf{x}}_{\mathbf{a}}(t)\right]+a_{0} \mathscr{H}_{2}(s) \mathscr{L}[\ddot{\mathbf{x}}(t)] \\
& \mathscr{L}[\phi(t)]=a_{1} \mathscr{H}_{2}(s) \mathscr{L}[\ddot{\mathbf{u}}(t)]+\mathscr{H}_{1}(s) \mathscr{L}[\ddot{\mathbf{u}}(t)],
\end{aligned}
$$

where

$$
\begin{aligned}
& \mathscr{H}_{1}(s)=\delta s G_{5}(s), \\
& \mathscr{H}_{2}(s)=\delta s^{2} G_{5}(s), \\
& \mathscr{H}_{3}(s)=\delta s^{3} G_{5}(s)
\end{aligned}
$$

Remark 2. The filters $\mathscr{H}_{i}(s), i=1,2,3$, are band pass filters, and they are designed to encompass the frequency band of the seismically excited building and to attenuate low and high frequency measurement noise.
TABLE 1: Boundary conditions for a shear building.

\begin{tabular}{lc}
\hline Boundary & $\begin{array}{c}\text { Absolute acceleration } \\
\ddot{x}_{a}(t)=\ddot{x}_{(}(t)+\ddot{x}_{g}(t)\end{array}$ \\
\hline$h=0$ & $\ddot{x}_{a}(0, t)=\ddot{x}_{g}(t)$ \\
& $\ddot{x}_{a}^{\prime}(0, t)=0$ \\
$h=H$ & $\ddot{x}_{a}^{\prime \prime}(H, t)=0$ \\
& $\ddot{x}_{a}^{\prime \prime \prime}(H, t)=0$ \\
\hline
\end{tabular}

\section{Estimation of the Responses at Noninstrumented Floor Levels}

In practice, most of the buildings are instrumented in only some floors; it means that not all signals $\ddot{x}_{a_{1}}(t), \ldots, \ddot{x}_{a_{n}}(t)$, which appear in variables $\ddot{\mathbf{x}}(t), \ddot{\mathbf{x}}_{\mathbf{a}}(t)$, and $\ddot{\mathbf{u}}(t)$ of parameterization (22), are available. In order to implement $\ddot{\mathbf{x}}(t), \ddot{\mathbf{x}}_{\mathbf{a}}(t)$ and $\ddot{\mathbf{u}}(t)$, the acceleration types at noninstrumented floors are reconstructed by means of cubic spline shape functions, which are described in this section.

Consider a building with height $H$ and $n$ stories that is instrumented at its basement and at $P$ floors, as shown in Figure 1. Moreover, let $h_{0}=0$ and $r_{0}(t)=\ddot{x}_{g}(t)$ be the height and acceleration response at the basement, respectively. Similarly, terms $h_{p}$ and $r_{p}(t), p=1,2, \ldots, P$, are the height and absolute acceleration at the $p$ th instrumented floor, respectively, where $r_{p}(t) \in \ddot{\mathbf{x}}_{\mathbf{a}}(t)$.

Let $\ddot{x}_{a}(h, t)$ be the absolute acceleration of the building at height $h$. Using (3) yields $\ddot{x}_{a}(0, t)=\ddot{x}_{g}(t)$ and $\ddot{x}_{a}(H, t)=$ $\ddot{x}_{n a}(t)$. In addition, let $h_{*}$ be the height of the $w$ th noninstrumented floor that is located within the subinterval $\Delta_{p}=h_{p+1}-h_{p}$ delimited by two instrumented floors with heights $h_{p}$ and $h_{p+1}$, where $h_{p+1}>h_{p}$. The response at this noninstrumented floor is given by $\ddot{x}_{a}\left(h_{*}, t\right)$, or, equivalently, $\ddot{x}_{a}\left(h_{*}, t\right)=\ddot{x}_{a_{w}}(t)$ according to definition (3). An estimate $\widehat{\ddot{x}}_{a}\left(h_{*}, t\right)=\widehat{\ddot{x}}_{a_{w}}(t)$ of $\ddot{x}_{a}\left(h_{*}, t\right)=\ddot{x}_{w a}(t)$ is computed through the following cubic spline shape function:

$$
\begin{aligned}
\widehat{\ddot{x}}_{a_{w}}(t)= & \widehat{\ddot{x}}_{a}\left(h_{*}, t\right) \\
= & a_{p}(t)+b_{p}(t)\left[h_{*}-h_{p}\right]+d_{p}\left[h_{*}-h_{p}\right]^{2} \\
& +e_{p}(t)\left[h_{*}-h_{p}\right]^{3}, \quad h_{*} \in\left[h_{p}, h_{p+1}\right],
\end{aligned}
$$

where $a_{p}(t), b_{p}(t), d_{p}(t)$, and $e_{p}(t)$ are the coefficients of the $p$ th cubic polynomial, which are computed at every sampling instant from continuity of the spline function, from responses $r_{p}(t), p=0,1,2, \ldots, P$, at the instrumented floors, and from the boundary conditions of the absolute acceleration of the building. These conditions assume that the building behaves as a cantilever, and they are shown in Table 1, where superscripts ', ", and '"' indicate the first, second, and third derivative with respect to the spatial variable $h$. Appendices $A$ and $B$ present the cubic spline function obtained if the acceleration response at the top floor is available or not, respectively.

Once absolute acceleration $\widehat{\ddot{x}}_{a_{w}}(t)$ of the $w$ th noninstrumented floor has been obtained, it is possible to estimate its 


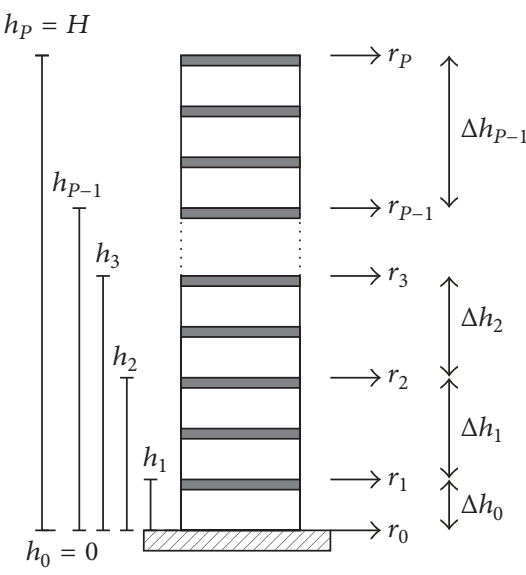

(a) Building instrumented at the top floor

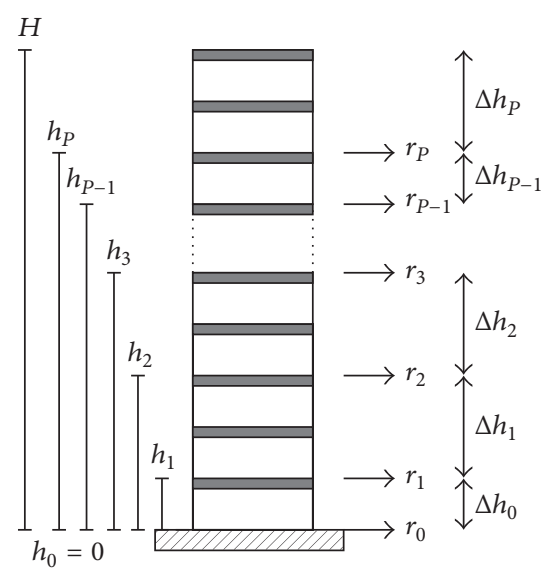

(b) Building noninstrumented at the top floor

Figure 1: Building with height $H$ instrumented in $P$ floor levels.

relative acceleration $\widehat{\ddot{x}}_{w}(t)$ through the expression $\widehat{\ddot{x}}_{w}(t)=$ $\widehat{\ddot{x}}_{a_{w}}(t)-\ddot{x}_{g}(t)$. Then, the unavailable responses $\ddot{x}_{w}(t)$ and $\ddot{x}_{a_{w}}(t)$ in variables $\ddot{\mathbf{x}}(t), \ddot{\mathbf{x}}_{\mathbf{a}}(t)$ and $\ddot{\mathbf{u}}(t)$ are replaced by their estimates $\widehat{\ddot{x}}_{w}(t)$ and $\widehat{\ddot{x}}_{a_{w}}(t)$, respectively. From now on, variables constructed with recorded and reconstructed responses are denoted as $\widehat{\ddot{\mathbf{x}}}_{\mathbf{a}}(t), \widehat{\hat{\mathbf{x}}}(t)$, and $\widehat{\overrightarrow{\mathbf{u}}}(t)$, which are given by

$$
\begin{aligned}
\widehat{\ddot{\mathbf{x}}}(t) & =\ddot{\mathbf{x}}(t)+\boldsymbol{\varepsilon}(t) \\
\widehat{\ddot{\mathbf{x}}}_{\mathbf{a}}(t) & =\ddot{\mathbf{x}}_{\mathbf{a}}(t)+\boldsymbol{\varepsilon}_{\mathbf{a}}(t) \\
\widehat{\ddot{\mathbf{u}}}(t) & =\ddot{\mathbf{u}}(t)+\boldsymbol{\varepsilon}_{\mathbf{u}}(t),
\end{aligned}
$$

where terms $\boldsymbol{\varepsilon}(t), \boldsymbol{\varepsilon}_{\boldsymbol{a}}(t)$, and $\boldsymbol{\varepsilon}_{\mathbf{u}}(t)$ depend on the error of the reconstructed responses and on the noise of the recorded responses.

\section{Parameter Estimation of the Building}

Substituting signals $\widehat{\ddot{\mathbf{x}}}(t), \widehat{\ddot{\mathbf{x}}}_{a}(t)$, and $\widehat{\overrightarrow{\mathbf{u}}}(t)$ given in (28) into (22)-(24) yields

$$
\widehat{\chi}(t)=\widehat{\phi}(t) \boldsymbol{\theta}+I_{5}[\boldsymbol{\lambda}(t)]
$$

where variables $\widehat{\chi}(t)$ and $\widehat{\phi}(t)$ have the same structure as $\chi(t)$ and $\phi(t)$, respectively. However, $\widehat{\chi}(t)$ and $\hat{\phi}(t)$ contain the terms $\widehat{\hat{\mathbf{x}}}_{\mathbf{a}}(t), \widehat{\hat{\mathbf{x}}}(t)$, and $\widehat{\ddot{\mathbf{u}}}(t)$ instead of $\ddot{\mathbf{x}}_{\mathbf{a}}(t), \ddot{\mathbf{x}}(t)$, and $\ddot{\mathbf{u}}(t)$. On the other hand, vector $\boldsymbol{\lambda}(t)$ depends on $\boldsymbol{\varepsilon}, \boldsymbol{\varepsilon}_{\mathbf{a}}$, and $\boldsymbol{\varepsilon}_{\mathbf{u}}$. The Laplace transform of $\boldsymbol{\lambda}(t)$ is given by

$$
\begin{aligned}
\boldsymbol{\Lambda}(s)= & \mathscr{L}[\boldsymbol{\lambda}(t)] \\
= & s^{3} \mathscr{L}\left[\boldsymbol{\varepsilon}_{\mathbf{a}}\right]+s^{2}\left(a_{0} \mathscr{L}[\boldsymbol{\varepsilon}]-a_{1} \mathscr{L}\left[\boldsymbol{\varepsilon}_{\mathbf{u}}\right] \boldsymbol{\theta}\right) \\
& -s \mathscr{L}\left[\boldsymbol{\varepsilon}_{\mathbf{u}}\right] \boldsymbol{\theta}
\end{aligned}
$$

Employing (20) and (30) allows obtaining the next Laplace transform of $I_{5}[\lambda(t)]$

$$
\begin{aligned}
\mathscr{L}\left[I_{5}[\lambda(t)]\right]= & \mathscr{H}_{3}(s) \mathscr{L}\left[\boldsymbol{\varepsilon}_{\mathbf{a}}\right] \\
& +\mathscr{H}_{2}(s)\left(a_{0} \mathscr{L}[\boldsymbol{\varepsilon}]-a_{1} \mathscr{L}\left[\boldsymbol{\varepsilon}_{\mathbf{u}}\right] \boldsymbol{\theta}\right) \\
& -\mathscr{H}_{1}(s) \mathscr{L}\left[\boldsymbol{\varepsilon}_{\mathbf{u}}\right] \boldsymbol{\theta},
\end{aligned}
$$

where $\mathscr{H}_{i}(s), i=1,2,3$, were previously defined in (26).

Expression (29) can be rewritten as

$$
\widehat{\chi}\left(k T_{s}\right)=\widehat{\phi}\left(k T_{s}\right) \boldsymbol{\theta}+I_{5}\left[\lambda\left(k T_{s}\right)\right]
$$

where $t=k T_{s}, k=0,1,2, \ldots$, are the sampling instants of signals $\widehat{\chi}(t)$ and $\widehat{\phi}(t)$. Omitting $T_{s}$ in (32) leads to

$$
\widehat{\chi}(k)=\widehat{\phi}(k) \boldsymbol{\theta}+I_{5}[\lambda(k)]
$$

In order to estimate the parameter vector $\boldsymbol{\theta}$ in (33), the Least Squares (LS) algorithm is employed, which is defined as [31]

$$
\begin{aligned}
\widehat{\boldsymbol{\theta}} & =\mathbf{P}(N) \sum_{k=0}^{N} \widehat{\boldsymbol{\phi}}^{T}(k) \widehat{\boldsymbol{\chi}}(k), \\
\mathbf{P}(N) & =\left[\sum_{k=0}^{N} \widehat{\boldsymbol{\phi}}^{T}(k) \hat{\boldsymbol{\phi}}(k)\right]^{-1},
\end{aligned}
$$

where $\mathbf{P}$ is the covariance matrix; $N$ is the number of samples of $\hat{\boldsymbol{\chi}}$ and $\widehat{\phi}$. Note that vector $\widehat{\boldsymbol{\theta}}$ can be computed only if matrix $\mathbf{P}$ exists.

On the other hand, $\boldsymbol{\theta}$ can also be recursively identified since the responses at noninstrumented floor levels can be 
computed at every sampling period $T_{s}$. The recursive version of the LS, denoted as RLS, is given by

$$
\widehat{\boldsymbol{\theta}}(k)=\widehat{\boldsymbol{\theta}}(k-1)+\mathbf{L}(k) \boldsymbol{\epsilon}(k)
$$

$\mathbf{L}(k)$

$$
\begin{aligned}
& =\mathbf{P}(k-1) \widehat{\phi}^{T}(k)\left[\beta \mathbf{I}_{n \times n}+\widehat{\phi}(k) \mathbf{P}(k-1) \widehat{\phi}(k)^{T}\right]^{-1} \\
& \mathbf{P}(k)=\frac{[\mathbf{P}(k-1)-\mathbf{L}(k) \widehat{\phi}(k) \mathbf{P}(k-1)]}{\beta} \\
& \boldsymbol{\epsilon}(k)=\hat{\chi}(k)-\widehat{\phi}(k) \hat{\boldsymbol{\theta}}(k),
\end{aligned}
$$

where $\beta$ is the forgetting factor such that $0<\beta \leq 1$; moreover, $\boldsymbol{\epsilon}(k)$ is the output estimation error.

Proposition 3. Suppose that input signal $\ddot{x}_{g}(t)$ is persistently exciting at least of order $\mathfrak{b}=2 n-1$, where $\mathfrak{b}$ is the number of parameters of $\boldsymbol{\theta}$ to be estimated, then vector norm $\|\tilde{\boldsymbol{\theta}}\|$ of the parameter estimation error $\widetilde{\boldsymbol{\theta}}$, defined as $\widetilde{\boldsymbol{\theta}}=\boldsymbol{\theta}-\widehat{\boldsymbol{\theta}}$, is bounded. Moreover, the smaller the perturbation term $I_{5}\left[\lambda\left(k T_{s}\right)\right]$ in (33), the smaller the value of $\|\widetilde{\boldsymbol{\theta}}\|$.

Proof. It is given in books $[31,32]$.

5.1. Estimation of Modal Parameters. Once that vector $\widehat{\boldsymbol{\theta}}$ has been estimated with the offline or the online estimation methodology, it is possible to identify the natural frequencies and modal damping factors of the building. To this end, the following matrix $\widehat{\mathbf{A}}$, which is an estimate of matrix $\mathbf{A}$ given in (8), is constructed:

$$
\begin{aligned}
\widehat{\mathbf{A}} & =\left[\begin{array}{ccc}
\mathbf{O}_{\mathbf{n} \times \mathbf{n}} & \mathbf{I}_{\mathbf{n} \times \mathbf{n}} \\
-\widehat{\mathbf{M}^{-1} \mathbf{K}} & -\widehat{\mathbf{M}^{-1} \mathbf{C}}
\end{array}\right] \\
\widehat{\mathbf{M}^{-1} \mathbf{K}} & =\left[\begin{array}{cccc}
\widehat{\theta}_{1}+\widehat{\theta}_{2} & -\widehat{\theta}_{2} & \cdots & 0 \\
-\widehat{\theta}_{3} & \widehat{\theta}_{3}+\widehat{\theta}_{4} & \cdots & 0 \\
\vdots & \vdots & \ddots & \vdots \\
0 & 0 & \cdots & \widehat{\theta}_{2 n-1}
\end{array}\right] \\
\widehat{\mathbf{M}^{-1} \mathbf{C}} & =a_{0} \mathbf{I}_{\mathbf{n} \times \mathbf{n}}+a_{1} \widehat{\mathbf{M}^{-1} \mathbf{K}},
\end{aligned}
$$

where parameters $\widehat{\theta}_{i}, i=1,2, \ldots, 2 n-1$, are the entries of vector $\widehat{\boldsymbol{\theta}}$. Note that (38) is deduced from (5).

The eigenvalues of the matrix $\widehat{\mathbf{A}}$ in (36) are given by

$$
\begin{aligned}
& \lambda_{i, 1}=-\sigma_{i}+j \omega_{i}=-\widehat{\zeta}_{i} \widehat{\omega}_{i}+j \widehat{\omega}_{i} \sqrt{1-\widehat{\zeta}^{2}}, \\
& \lambda_{i, 2}=-\sigma_{i}-j \omega_{i}=-\widehat{\zeta}_{i} \widehat{\omega}_{i}-j \widehat{\omega}_{i} \sqrt{1-\widehat{\zeta}^{2}},
\end{aligned}
$$

where $\sigma_{i}=\widehat{\zeta}_{i} \widehat{\omega}_{i}$ and $\widehat{\omega}_{i}=\widehat{\omega}_{i} \sqrt{1-\widehat{\zeta}^{2}}$. Moreover, $\widehat{\omega}_{i}$ and $\widehat{\zeta}_{i}$, $i=1,2, \ldots, n$, are, respectively, the estimates of the natural frequency $\omega_{i}$ and damping factor $\zeta_{i}$ corresponding to the $i$ th mode of building model (1).
From (39), the following equations for computing the parameters $\widehat{\omega}_{i}$ and $\widehat{\zeta}_{i}$ are obtained:

$$
\begin{aligned}
& \widehat{\omega}_{i}=\sqrt{\sigma_{i}^{2}+\omega_{i}^{2}}, \\
& \widehat{\zeta}_{i}=\frac{\sigma_{i}}{\widehat{\omega}_{i}},
\end{aligned}
$$

$$
i=1,2, \ldots, n
$$

5.2. Estimation of Matrices $M, K$, and $C$. Assume that mass $m_{1}$ of the building is known; then matrix $\widehat{\mathbf{C}}$ and the entries of matrices $\widehat{\mathbf{M}}$ and $\widehat{\mathbf{K}}$ are given by

$$
\begin{aligned}
& \widehat{k}_{1}=\widehat{m}_{1} \widehat{\theta}_{1}, \\
& \widehat{k}_{i}=\widehat{m}_{i-1} \widehat{\theta}_{(2 i-2)}, \\
& \widehat{m}_{i}=\frac{\widehat{k}_{i}}{\widehat{\theta}_{(2 i-1)}}, \quad i=2,3, \ldots, n \\
& \widehat{\mathbf{C}}=a_{0} \widehat{\mathbf{M}}+a_{1} \widehat{\mathbf{K}}
\end{aligned}
$$

A similar procedure can be carried out if another floor mass is known instead of $m_{1}$.

\section{High Gain State Observer}

The proposed high gain state observer employs the building model estimated by the LS method. This observer estimates the complete state of a building instrumented at only few floor levels, and it is given by

$$
\begin{aligned}
\dot{\hat{\boldsymbol{\eta}}}(t) & =\widehat{\mathbf{A}} \widehat{\boldsymbol{\eta}}(t)+\mathbf{B} \ddot{x}_{g}(t)+\mathbf{L} \widetilde{\psi}(t), \\
\widetilde{\psi}(t) & =\widehat{\ddot{\mathbf{x}}}_{\mathbf{a}}(t)-\widehat{\mathbf{y}}_{\mathbf{o}}(t) \\
\widehat{\ddot{\mathbf{x}}}_{\mathbf{a}}(t) & =\ddot{\mathbf{x}}_{\mathbf{a}}(t)+\boldsymbol{\varepsilon}_{\mathbf{a}}(t)=\mathbf{D} \boldsymbol{\eta}(t)+\boldsymbol{\varepsilon}_{\mathbf{a}}(t), \\
\widehat{\mathbf{y}}_{\mathbf{o}}(t) & =\widehat{\mathbf{D}} \widehat{\boldsymbol{\eta}}(t) \\
\widehat{\boldsymbol{\eta}} & =\left[\widehat{\mathbf{x}}^{\mathbf{T}}, \hat{\dot{\mathbf{x}}}^{\mathbf{T}}\right]^{T}=\left[\widehat{x}_{1}, \widehat{x}_{2}, \ldots, \widehat{x}_{n}, \hat{\dot{x}}_{1}, \widehat{\dot{x}}_{2}, \ldots, \hat{\dot{x}}_{n}\right]^{T},
\end{aligned}
$$

where $\widehat{\boldsymbol{\eta}}$ is an estimate of $\boldsymbol{\eta}, \mathbf{L}$ is the observer gain matrix, variable $\widehat{\ddot{\mathbf{x}}}_{\mathbf{a}}$ is established in (28), term $\widehat{\mathbf{y}}_{\mathbf{o}}$ is the absolute acceleration estimated by the observer, matrix $\widehat{\mathbf{A}}$ is presented in (36), and matrix $\widehat{\mathbf{D}}$, which is an estimate of $\mathbf{D}$ in (10), is defined as

$$
\widehat{\mathbf{D}}=\left[\widehat{\mathbf{M}^{-1} \mathbf{K}}, \widehat{\mathbf{M}^{-1} \mathrm{C}}\right]
$$

with $\widehat{\mathbf{M}^{-1} \mathbf{K}}$ and $\widehat{\mathbf{M}^{-1} \mathbf{C}}$ shown in (37) and (38), respectively.

Define the state estimation error $\widetilde{\boldsymbol{\eta}}(t)$ as follows:

$$
\begin{aligned}
\widetilde{\boldsymbol{\eta}}(t) & =\boldsymbol{\eta}(t)-\widehat{\boldsymbol{\eta}}(t)=\left[\begin{array}{c}
\mathbf{x}(t) \\
\dot{\mathbf{x}}(t)
\end{array}\right]-\left[\begin{array}{c}
\widehat{\mathbf{x}}(t) \\
\hat{\dot{\mathbf{x}}}(t)
\end{array}\right]=\left[\begin{array}{c}
\widetilde{\mathbf{x}}(t) \\
\tilde{\dot{\mathbf{x}}}(t)
\end{array}\right] \\
& =\left[\widetilde{x}_{1}, \widetilde{x}_{2}, \ldots, \widetilde{x}_{n}, \tilde{\dot{x}}_{1}, \tilde{\dot{x}}_{2}, \ldots, \tilde{\dot{x}}_{n}\right]^{T}
\end{aligned}
$$


Then, the state estimation error dynamics $\dot{\overrightarrow{\boldsymbol{\eta}}}(t)$ is given by

$$
\begin{aligned}
\dot{\overrightarrow{\boldsymbol{\eta}}}(t)= & \dot{\boldsymbol{\eta}}(t)-\dot{\hat{\boldsymbol{\eta}}}(t) \\
= & (\mathbf{A}-\mathbf{L D}) \boldsymbol{\eta}(t)+(\widehat{\mathbf{A}}-\mathbf{L} \widehat{\mathbf{D}}) \widetilde{\boldsymbol{\eta}}(t)-\mathbf{L} \boldsymbol{\varepsilon}_{\mathbf{a}}(t) \\
= & (\mathbf{A}-\mathbf{L D}) \boldsymbol{\eta}(t)+(\widehat{\mathbf{A}}-\mathbf{L} \widehat{\mathbf{D}})(\boldsymbol{\eta}(t)-\widehat{\boldsymbol{\eta}}(t)) \\
& -\mathbf{L} \boldsymbol{\varepsilon}_{\mathbf{a}}(t)=(\widehat{\mathbf{A}}-\mathbf{L} \widehat{\mathbf{D}}) \widetilde{\boldsymbol{\eta}}(t)+\boldsymbol{\Xi}(t) \\
= & \mathbf{A}_{*} \widetilde{\boldsymbol{\eta}}(t)+\boldsymbol{\Xi}(t)
\end{aligned}
$$

with

$$
\begin{aligned}
\mathbf{A}_{*} & =(\widehat{\mathbf{A}}-\mathbf{L} \widehat{\mathbf{D}}), \\
\Xi(t) & =(\widetilde{\mathbf{A}}-\mathbf{L} \widetilde{\mathbf{D}}) \boldsymbol{\eta}(t)-\mathbf{L} \boldsymbol{\varepsilon}_{\mathbf{a}}(t) \\
\widetilde{\mathbf{A}} & =\mathbf{A}-\widehat{\mathbf{A}}, \\
\widetilde{\mathbf{D}} & =\mathbf{D}-\widehat{\mathbf{D}}
\end{aligned}
$$

Proposition 4. Let $\mathbf{L}$ be the gain of the state observer defined as

$$
\mathbf{L}=\left[\begin{array}{ll}
\mathbf{O}_{\mathbf{n} \times \mathbf{n}} & -\gamma \mathbf{I}_{\mathbf{n} \times \mathbf{n}}
\end{array}\right]^{T}
$$

(1) If $\gamma$ satisfies

$0 \leq \gamma \leq \kappa=\min \left[\varrho_{1}, \varrho_{2}, \ldots, \varrho_{n}\right]$,

$\varrho_{i}=\frac{1}{\widehat{\zeta}_{i}^{2}}-1$,

$$
i=1,2, \ldots, n,
$$

where $\hat{\zeta}_{i}$ is presented in (40), and $\min [\cdot]$ denotes the smallest value of the set [·], then, one has the following:

(a) The eigenvalues of matrix $\mathbf{A}_{*}$ in (48) are given by

$$
\begin{aligned}
& \lambda_{i, 1}^{*}=-\sigma_{i}^{*}+j \omega_{i}^{*}, \\
& \lambda_{i, 2}^{*}=-\sigma_{i}^{*}-j \omega_{i}^{*} \\
& \sigma_{i}^{*}=\zeta_{i}^{*} \omega_{i}^{*} \\
& \omega_{i}^{*}=\omega_{i}^{*} \sqrt{1-\zeta^{* 2}} \\
& \omega_{i}^{*}=\widehat{\omega}_{i} \sqrt{1+\gamma}, \\
& \zeta_{i}^{*}=\widehat{\zeta}_{i} \sqrt{1+\gamma},
\end{aligned}
$$

where $i=1,2, \ldots, n$ and $\widehat{\omega}_{i}$ and $\widehat{\zeta}_{i}$ are the natural frequency and damping factor of the ith mode of the estimated building model, which are computed using (40).

(b) Increasing the value of gain $\gamma$ in (51)-(52) allows the real part of the eigenvalues of matrix $\mathbf{A}_{*}$ to be more negative. (c) Norm of the estimation error $\widetilde{\boldsymbol{\eta}}$ is bounded, and when $t \longrightarrow \infty$ it satisfies

$$
\begin{aligned}
\|\widehat{\boldsymbol{\eta}}(t)\| & \leq \frac{\mu}{\widehat{\zeta}_{\bullet} \widehat{\omega} \cdot}\|\boldsymbol{E}\|\|\boldsymbol{\eta}(t)\|+\frac{\mu \gamma\left\|\boldsymbol{\varepsilon}_{\mathbf{a}}(t)\right\|}{(1+\gamma) \widehat{\zeta}_{.} \widehat{\omega} .} \\
\boldsymbol{E} & =\left[\begin{array}{cc}
\mathbf{O}_{\mathbf{n} \times \mathbf{n}} & \frac{1}{1+\gamma} \mathbf{I}_{\mathbf{n} \times \mathbf{n}} \\
-\widehat{\mathbf{M}}^{-\mathbf{1}} \mathbf{K} & -\frac{\mathbf{M}^{-1} \mathbf{C}}{}
\end{array}\right],
\end{aligned}
$$$$
\widetilde{\mathbf{M}^{-1} \mathbf{K}}=\mathbf{M}^{-1} \mathbf{K}-\widehat{\mathbf{M}^{-1} \mathbf{K}},
$$$$
\widetilde{M^{-1} \mathbf{C}}=\mathbf{M}^{-1} \mathrm{C}-\widehat{\mathbf{M}^{-1} \mathrm{C}}
$$$$
\mu=\|\mathfrak{M}\|\left\|\mathfrak{M}^{-1}\right\|,
$$

$\widehat{\zeta}_{.} \widehat{\omega}_{\bullet}=\min _{1 \leq i \leq n}\left\{\widehat{\zeta}_{i} \widehat{\omega}_{i}\right\}$

where $\mathbf{M}$ is a matrix, whose columns are the eigenvectors of matrix $\mathbf{A}_{*}$.

(2) If $\gamma \longrightarrow \infty$, then the eigenvalues of $A_{*}$ approach $\lambda_{i, 1}^{*}=$ $-\widehat{\omega}_{i} /\left(2 \widehat{\zeta}_{i}\right)$ and $\lambda_{i, 2}^{*}=-\infty, i=1,2, \ldots, n$.

Proof. See Appendix C.

6.1. Attenuation of the State Estimation Error. The norm $\|\widehat{\boldsymbol{\eta}}(t)\|$ of the state estimation error can be reduced as follows:

(i) Increase $\gamma$ in order to decrease $\|\mathfrak{E}\|$ and to obtain a fast response for the observer. Based on our experience, good results in the state estimation are obtained using a gain $\gamma$ between $\kappa / 10$ and $\kappa$, where $\kappa$ was defined in (52).

(ii) Reduce $\left\|\widetilde{\mathbf{M}^{-1} \mathbf{K}}\right\|$ and $\left\|\widetilde{\mathbf{M}^{-1} \mathbf{C}}\right\|$, which allows decreasing $\|\mathfrak{E}\|$. According to Proposition 3, the smaller the term $I_{5}[\lambda]$ in (33) is, the smaller the parametric error norms $\left\|\widetilde{\mathbf{M}^{-1} \mathbf{K}}\right\|$ and $\left\|\widetilde{\mathbf{M}^{-1} \mathbf{C}}\right\|$ are. Signal $I_{5}[\lambda]$ is filtered through the filters $\mathscr{H}_{1}(s), \mathscr{H}_{2}(s)$, and $\mathscr{H}_{3}(s)$ shown in (31). They are designed to include the bandwidth of the building responses and to filter measurement noise, which in turn attenuates the term $I_{5}[\lambda]$ and norms $\left\|\widetilde{\mathbf{M}^{-1} \mathbf{K}}\right\|$ and $\left\|\widetilde{\mathbf{M}^{-1} \mathbf{C}}\right\|$.

(iii) Instrument the building at regular intervals over its height, which permits reducing $\left\|\boldsymbol{\varepsilon}_{\mathbf{a}}\right\|[19,33]$. These references also show that increasing the total number of instrumented floors decreases the value of $\left\|\boldsymbol{\varepsilon}_{\mathbf{a}}\right\|$.

(iv) Attenuate the measurement noise corrupting the state observer. To achieve this, vector $\widehat{\ddot{\mathbf{x}}}_{\mathbf{a}}(t)$ in (28) is replaced by the following filtered vector $\widehat{\ddot{\mathbf{x}}}_{\mathbf{a f}}(t)$ in variable $\widetilde{\psi}$ of the state observer (42):

$$
\widehat{\ddot{\mathbf{x}}}_{\mathrm{af}}(t)=\mathscr{L}^{-1}\left[\mathfrak{F}_{1}(s)\right] * \widehat{\ddot{\mathbf{x}}}_{\mathrm{a}}(t),
$$

where $*$ is the convolution operator, and $\mathfrak{F}_{1}(s)$ is a fourth-order low-pass Butterworth filter, whose cutoff frequency is appropriately chosen. 


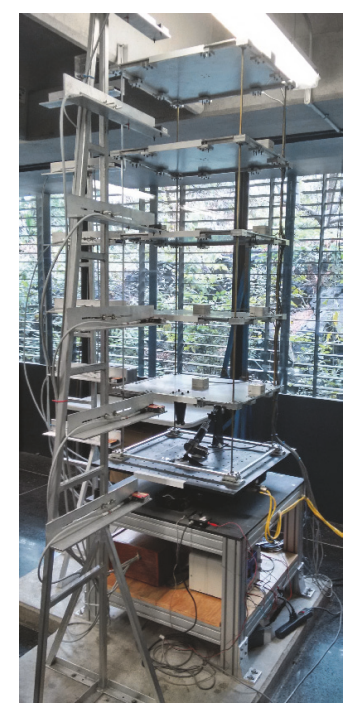

FIGURE 2: Experimental structure.

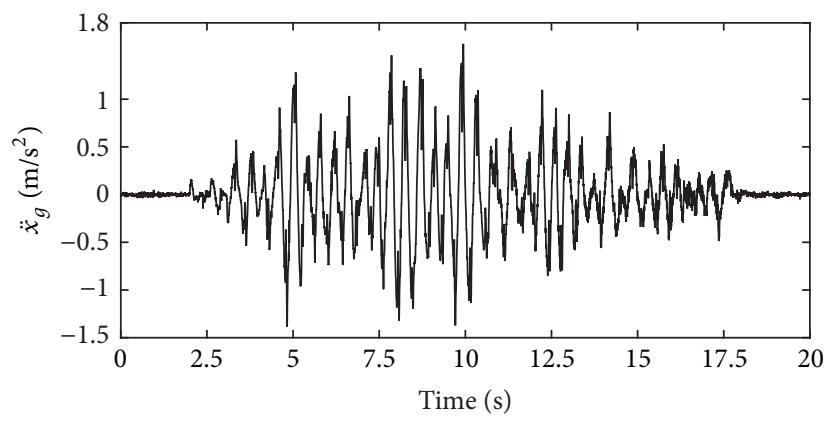

Figure 3: Excitation of the experimental structure.

\section{Experimental Results}

Figure 2 depicts a five-story small scale building with dimensions $65 \times 55 \times 175.5 \mathrm{~cm}$, which is used during the experiments. The structure is mounted over a shake table with Parker linear motors 406T03LXR, and it is built with aluminum with exception of a column of each floor, which is made from brass. During the experiments, the structure is excited with the North-South component of the Mexico City 1985 earthquake, which is fitted in amplitude to be in agreement with the structure and shown in Figure 3. The responses of the shake table and floors are measured through Analog Devices accelerometers, model ADXL203, placed at every floor and at the base. Moreover, the absolute position of each floors is obtained by means of Micro-Epsilon laser sensors, model optoNCDT 1302. These sensors are only used for comparing the displacements and velocities of the floors with their estimates provided by the high gain state observer. Filtering the response of the laser sensors with the filter $(75 \pi)^{2} s /(s+$ $75 \pi)^{2}$ produces the nominal velocity of the building floors. Filter $\mathfrak{\mho}_{1}(s)$ in (58) has a cut-off frequency of $100 \mathrm{~Hz}$. The parameter identification algorithm and the state observer are implemented in Matlab-Simulink. Data acquisition is carried out through two National Instruments PCI-6221 cards, which communicate with a personal computer by means of the
Simulink Desktop Real-Time toolbox. It is worth mentioning that a sampling period of $1 \mathrm{~ms}$ is used during the experiments.

The first two natural frequencies of the small scale building are $\omega_{1}=11.07$ and $\omega_{2}=31.8 \mathrm{rad} / \mathrm{s}$, which were obtained from frequency response experiments. It is considered that the first two modes of the structure have a damping factor of $1 \%$, i.e., $\zeta_{1}=\zeta_{2}=0.01$, based on experimental data and since it is slightly damped. During experiments we have observed that $\zeta_{1}$ and $\zeta_{2}$ vary between $0.7 \%$ and $1.6 \%$, and this variation depends on the excitation signal. In order to compute the coefficients $a_{0}$ and $a_{1}$ of the Rayleigh damping, we decided to fix both damping ratios $\zeta_{1}$ and $\zeta_{2}$ to $1 \%$, since this value is between $0.7 \%$ and $1.6 \%$. These natural frequencies and damping factors allow obtaining parameters $a_{1}=0.1642$ and $a_{2}=0.0005$ using (6), which are employed for computing the variables $\widehat{\chi}$ and $\widehat{\phi}$ of parameterization (33).

In order to determine the effectiveness of the cubic spline function in the response reconstruction of the $w$ th noninstrumented floor, the following function $\mathscr{E}_{w}$ is computed, which was previously defined in [19]

$$
\mathscr{E}_{w}=\sqrt{\frac{\sum_{k=0}^{P}\left[\ddot{x}_{a_{w}}(k)-\widehat{\ddot{x}}_{a_{w}}(k)\right]^{2}}{\sum_{k=0}^{N} \ddot{x}_{a_{w}}^{2}(k)}},
$$

where $\ddot{x}_{w a}$ and $\widehat{\ddot{x}}_{w a}$ are the nominal and estimated absolute acceleration of the $w$ th building floor, respectively. Value of function $\mathscr{E}_{w}$ depends on the number of instrumented floors and on their distribution along the building height. Let $\Pi_{j}^{P}, j=1,2, \ldots, l ; \iota=(n ! /[P !(n-P) !])$, be all possible distributions of $P$ instrumented floors of a building with $n$ stories. Each distribution produces a set of errors $\mathscr{E}_{w}$, and the mean value of these errors is denoted as $\overline{\mathscr{E}}$. It is considered that the smaller the value of $\overline{\mathscr{E}}$, the better the performance of the cubic spline functions.

On the other hand, let $\widetilde{\omega}_{i}$ be the identification error in percentage (\%) of the $i$ th natural frequency $\widehat{\omega}_{i}$ corresponding to estimated building model. Performance index $E_{\widetilde{\omega}}$ in (60) measures the quality of the identified model by taking into account all errors $\widetilde{\omega}_{i}$; i.e.,

$$
\begin{aligned}
& E_{\widetilde{\omega}}(\delta)=\sqrt{\sum_{i=1}^{5} \widetilde{\omega}_{i}^{2},} \\
& \widetilde{\omega}_{i}(\%)=\frac{\left|\widehat{\omega}_{i}-\omega_{i}\right|}{\omega_{i}} \times 100
\end{aligned}
$$

The smaller the value of $E_{\widetilde{\omega}}$, the better the quality of the model. Since function $E_{\widetilde{\omega}}$ depends on the integration period $\delta$ of the LIF, it is useful to compute the following terms:

$$
\begin{aligned}
E_{\widetilde{\omega}}^{*} & =\underset{\delta \in\left[\delta_{\min }, \delta_{\max }\right]}{\min } E_{\widetilde{\omega}}(\delta), \\
\delta^{*} & =\underset{\delta \in\left[\delta_{\min }, \delta_{\max }\right]}{\arg \min } E_{\widetilde{\omega}}(\delta), \\
f_{c}^{*} & =\frac{1}{\delta^{*}}
\end{aligned}
$$

The performance of the high gain state observer is also examined. To this end, function $E_{\widetilde{\mathbf{x}}}(\delta)$ in (62), which also depends on $\delta$, is computed. 
TABLE 2: Results obtained when all floors are instrumented.

\begin{tabular}{|c|c|c|c|c|c|c|c|c|c|}
\hline$\Pi_{j}^{5}$ & Instrumented floors & Non-instrumented floors & $\overline{\mathscr{E}}$ & $E_{\tilde{\omega}}^{*}$ & $\delta^{*}[\mathrm{~s}]$ & $f_{c}^{*}[\mathrm{~Hz}]$ & $E_{\tilde{\mathbf{x}}}^{\circ}$ & $\delta^{\circ}[\mathrm{s}]$ & $f_{c}^{\circ}[\mathrm{Hz}]$ \\
\hline 1 & 012345 & - & - & 1.96 & 0.035 & 28.57 & 6.37 & 0.055 & 18.18 \\
\hline
\end{tabular}

TABLE 3: Results with four instrumented floors.

\begin{tabular}{lccccccccc}
\hline$\Pi_{j}^{4}, j$ & Instrumented floors & Non-instrumented floors & $\overline{\mathscr{E}}$ & $E_{\widetilde{\omega}}^{*}$ & $\delta^{*}[\mathrm{~s}]$ & $f_{c}^{*}[\mathrm{~Hz}]$ & $E_{\tilde{\mathbf{x}}}^{\circ}$ & $\delta^{\circ}[\mathrm{s}]$ & $f_{c}^{\circ}[\mathrm{Hz}]$ \\
\hline 1 & 01245 & 3 & 0.18 & 3.50 & 0.04 & 25 & 11.64 & 0.048 & 20.83 \\
2 & 01345 & 2 & 0.27 & 3.59 & 0.038 & 26.32 & 9.45 & 0.058 \\
3 & 01235 & 4 & 0.16 & 4.18 & 0.04 & 25 & 13.38 & 0.048 & 20.83 \\
4 & 02345 & 1 & 0.45 & 5.79 & 0.044 & 22.73 & 7.50 & 0.062 & 16.13 \\
5 & 01234 & 5 & 0.32 & 12.32 & 0.068 & 14.71 & 26.07 & 0.048 & 20.83 \\
\hline
\end{tabular}

TABLE 4: Results with three instrumented floors.

\begin{tabular}{lccccccccc}
\hline$\Pi_{j}^{3}, j$ & Instrumented floors & Non-instrumented floors & $\overline{\mathscr{E}}$ & $E_{\tilde{\omega}}^{*}$ & $\delta^{*}[\mathrm{~s}]$ & $f_{c}^{*}[\mathrm{~Hz}]$ & $E_{\tilde{\mathbf{x}}}^{\circ}$ & $\delta^{\circ}[\mathrm{s}]$ & $f_{c}^{\circ}[\mathrm{Hz}]$ \\
\hline 1 & 0 & 235 & 13 & 0.48 & 5.17 & 0.046 & 21.74 & 13.88 & 0.054 \\
2 & 0245 & 14 & 0.68 & 5.21 & 0.048 & 20.83 & 11.98 & 0.05 & 20 \\
3 & 0235 & 34 & 0.63 & 6.08 & 0.046 & 21.74 & 14.63 & 0.056 & 17.86 \\
4 & 0125 & 25 & 0.53 & 8.55 & 0.054 & 18.52 & 25.4 & 0.048 & 20.83 \\
5 & 0134 & 35 & 0.61 & 9.03 & 0.05 & 20 & 26.27 & 0.046 & 21.74 \\
6 & 0124 & 23 & 0.55 & 9.98 & 0.05 & 20 & 21.83 & 0.044 & 22.73 \\
7 & 0145 & 15 & 0.74 & 10.98 & 0.052 & 19.23 & 18.71 & 0.038 & 26.32 \\
8 & 0234 & 12 & 0.78 & 13.15 & 0.07 & 14.29 & 25.88 & 0.05 \\
9 & 0345 & 45 & 0.98 & 14.57 & 0.06 & 16.67 & 12.71 & 0.064 & 15.63 \\
10 & 0123 & 0.96 & - & - & - & - & - \\
\hline
\end{tabular}

$$
\begin{aligned}
E_{\tilde{\mathbf{x}}}(\delta) & =\sum_{i=1}^{5}\left[\left\|\tilde{x}_{i}\right\|+\left\|\tilde{\dot{x}}_{i}\right\|\right], \\
\left\|\tilde{x}_{i}\right\| & =\sqrt{\sum_{j=1}^{N} \tilde{x}_{i}^{2}} \\
\left\|\tilde{\dot{x}}_{i}\right\| & =\sqrt{\sum_{j=1}^{N} \tilde{\dot{x}}_{i}^{2}}
\end{aligned}
$$

The better the reconstruction of the state, the smaller the value of $E_{\widetilde{\mathbf{x}}}(\delta)$. The following expressions depending on the minimum of function $E_{\widetilde{\mathbf{x}}}(\delta)$ are also computed during the experiments

$$
\begin{aligned}
E_{\widetilde{\mathbf{x}}}^{\circ} & =\min _{\delta \in\left[\delta_{\min }, \delta_{\max }\right]} E_{\widetilde{\mathbf{x}}}(\delta), \\
\delta^{\circ} & =\underset{\delta \in\left[\delta_{\min }, \delta_{\max }\right]}{\arg \min } E_{\widetilde{\mathbf{x}}}(\delta), \\
f_{c}^{\circ} & =\frac{1}{\delta^{\circ}}
\end{aligned}
$$

The goal of the experiments presented in the next subsection is to obtain and compare the value of the performance indexes defined above, when the small scale building is instrumented from one to five floors. A comparison of measured and estimated values of the state will also be included. In all the experiments, the value of the observer gain $\gamma$ is fixed to $\gamma=1000$.

7.1. Results with Five Instrumented Floors. This case represents the fully instrumented case, where no cubic splines functions are used, and it is included for comparison purposes. Table 2 shows the values of $E_{\widetilde{\omega}}^{*}, \delta^{*}, E_{\widetilde{\mathbf{x}}}^{\circ}$, and $\delta^{\circ}$ for the unique distribution of five instrumented floors, which is named $\Pi_{1}^{5}$. Moreover, Figures $4(\mathrm{a})$ and $4(\mathrm{~b})$ show, respectively, the variation of the performance indexes $E_{\widetilde{\omega}}(\delta)$ and $E_{\widetilde{\mathbf{x}}}(\delta)$, where $\delta \in[0.01,0.12]$. Note that $\delta^{*} \neq \delta^{\circ}$, indicating that the estimated model with the best quality does not produce the smallest value of $E_{\widetilde{\mathbf{x}}}(\delta)$. The reason is that term $\boldsymbol{\varepsilon}_{\mathrm{a}}$ in (43) that depends only on the measurement noise in this case with full instrumentation of the structure affects the performance of state observer, which provokes the fact that the identified model with the best quality does not originate the smallest value $E_{\widetilde{\mathbf{x}}}^{\circ}$. From Figures $4(\mathrm{a})$ and $4(\mathrm{~b})$, it is possible to see that $E_{\widetilde{\omega}}(\delta)$ varies within $[1.96,29.47]$, whereas function $E_{\widetilde{\mathbf{x}}}(\delta)$ takes values within the interval $[6.37,14.84]$.

7.2. Results with Four Instrumented Floors. Table 3 presents the parameters $\overline{\mathscr{E}}, E_{\widetilde{\omega}}^{*}, \delta^{*}, f_{c}^{*}, E_{\widetilde{\mathbf{x}}}^{\circ}, \delta^{\circ}$, and $f_{c}^{\circ}$, which are computed using the distributions $\Pi_{j}^{4}, j=1,2, \ldots, 5$. Furthermore, Figures 5(a) and 5(b) depict, respectively, the functions $E_{\widetilde{\omega}}$ and $E_{\widetilde{\mathbf{x}}}$ with respect to $\delta$. From these figures, it is possible to observe that values of $E_{\widetilde{\omega}}(\delta)$, produced by distributions $\Pi_{k}^{4}, j=1,2, \ldots, 4$, for $\delta \in[0.02,0.05]$, are similar. Moreover, functions $E_{\widetilde{\mathbf{x}}}(\delta)$ corresponding to these distributions have a small variation for $\delta \in[0.05,0.1]$. On the other hand, the smallest value of $\overline{\mathscr{E}}$ is obtained with distribution $\Pi_{3}^{4}$, but this layout does not produce the smallest values of $E_{\widetilde{\omega}}^{*}$ and $E_{\widetilde{\mathbf{x}}}^{\circ}$. Note that the largest values of $E_{\widetilde{\omega}}(\delta)$ and $E_{\widetilde{\mathbf{x}}}(\delta)$ for $\delta \in[0.02,0.06]$ are computed with distribution $\Pi_{5}^{4}$, i.e., where the top floor is not instrumented.

7.3. Results with Three Instrumented Floors. Table 4 presents the values of $\overline{\mathscr{E}}, E_{\widetilde{\omega}}^{*}, \delta^{*}, f_{c}^{*}, E_{\widetilde{\mathbf{x}}}^{\circ}, \delta^{\circ}$, and $f_{c}^{\circ}$ for distributions 


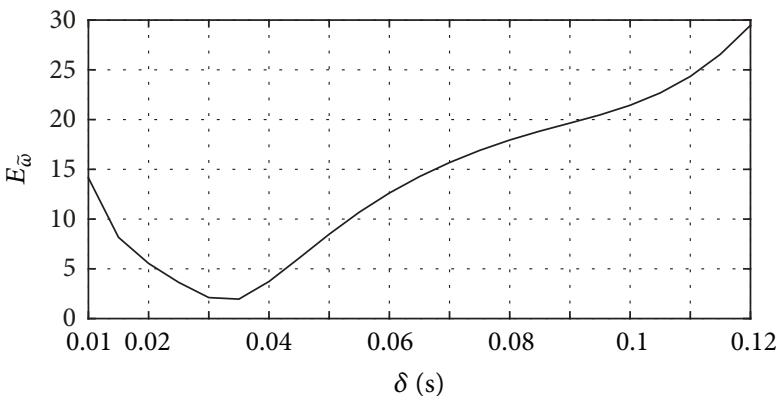

(a) $E_{\widetilde{\omega}}(\delta)$ versus $\delta$

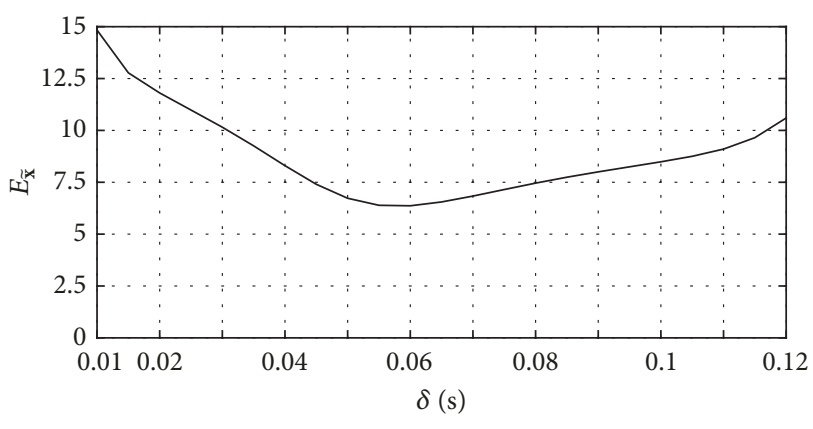

(b) $E_{\widetilde{\mathbf{x}}}(\delta)$ versus $\delta$

FIgURE 4: Functions $E_{\widetilde{\omega}}(\delta)$ and $E_{\widetilde{\mathbf{x}}}(\delta)$ computed when all the floors are instrumented.

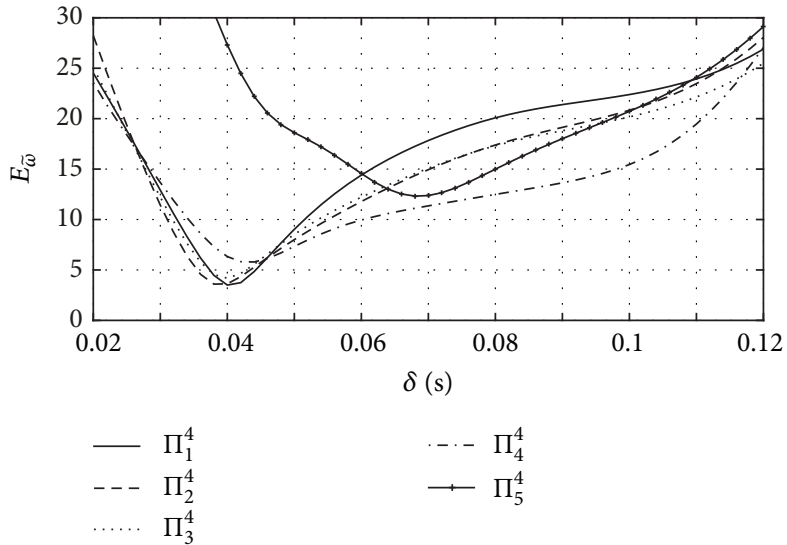

(a) $E_{\widetilde{\omega}}(\delta)$ versus $\delta$

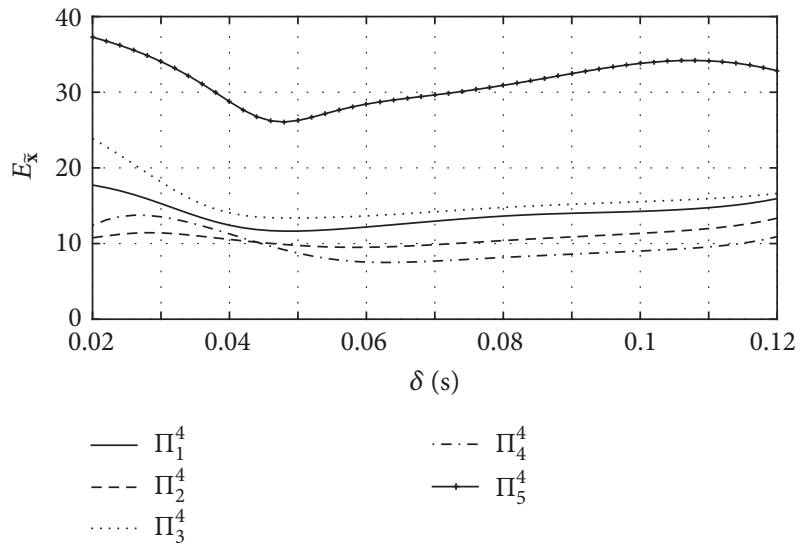

(b) $E_{\widetilde{\mathbf{x}}}(\delta)$ versus $\delta$

FIGURE 5: Functions $E_{\widetilde{\omega}}(\delta)$ and $E_{\widetilde{\mathbf{x}}}(\delta)$ computed with four instrumented floors.

$\Pi_{j}^{3}, j=1,2, \ldots, 9$, of three instrumented floors. It is not possible to identify the building model using layout $\Pi_{10}^{3}$, since the reconstructed absolute acceleration types at noninstrumented floors produce a regressor vector $\widehat{\phi}$ such that covariance matrices $\mathbf{P}$ in (34) and (35) do not exist. On the other side, Figures 6 and 7 show, respectively, the functions $E_{\widetilde{\omega}}$ and $E_{\widetilde{\mathbf{x}}}$ with respect to $\delta$. Note that distributions $\Pi_{j}^{3}, j=1,2,3$, which are uniform or approximately uniform along the building height, allow obtaining small values of $E_{\widetilde{\omega}}^{*}$ and $E_{\tilde{\mathrm{x}}}^{\circ}$, which are close to the ones computed with layouts $\Pi_{j}^{4}, j=1,2,3$, of four instrumented floors. On the other hand, distributions $\Pi_{k}^{3}, k=7,8,9$, that are not uniform over the building height produce the largest values of $\overline{\mathscr{E}}$ and $E_{\widetilde{\omega}}^{*}$; however, a small value of $E_{\widetilde{\mathbf{x}}}^{\circ}$ is computed with $\Pi_{9}^{3}$.

Figure 8 depicts the absolute acceleration types $\widehat{\ddot{x}}_{a_{2}}$ and $\widehat{\ddot{x}}_{a_{4}}$ and the two noninstrumented floors, which are reconstructed by the cubic shape function in the configuration $\Pi_{1}^{3}$. It is shown that these responses are close to their nominal value. On the other side, Figure 9 shows the entries $\widehat{\theta}_{i}, i=$ $1,2, \ldots, 9$, of the estimated vector $\widehat{\boldsymbol{\theta}}$ provided by the RLS and corresponding to layout $\Pi_{1}^{3}$. Note that parameters $\widehat{\theta}_{i}$, $i=1,2, \ldots, 9$, converge to a neighborhood around a constant value in approximately $5 \mathrm{~s}$.

7.4. Results with Two Instrumented Floors. Values of $\overline{\mathscr{E}}, E_{\widetilde{\omega}}^{*}$, $\delta^{*}, f_{c}^{*}, E_{\widetilde{\mathbf{x}}}^{\circ}, \delta^{\circ}$, and $f_{c}^{\circ}$ for distributions $\Pi_{j}^{2}, j=1,2, \ldots, 7$, are shown in Table 5. Furthermore, Figures 10 and 11 present, respectively, the performance indexes $E_{\widetilde{\omega}}$ and $E_{\widetilde{\mathbf{x}}}$ computed by varying $\delta$. Layout $\Pi_{1}^{2}$, with which the building is instrumented at approximately regular intervals, originates the smallest value of $E_{\widetilde{\omega}}^{*}$ and small values of $\overline{\mathscr{E}}$ and $E_{\widetilde{\mathbf{x}}}^{\circ}$ among the ones shown in Table 5. On the other hand, distributions $\Pi_{5}^{2}$ and $\Pi_{6}^{2}$ that also are approximately uniform along the building height produce large values of $E_{\widetilde{\omega}}^{*}$ but small values of $\overline{\mathscr{E}}$ and $E_{\tilde{\mathbf{x}}}^{\circ}$. It is worth mentioning that configurations $\Pi_{j}^{2}$, $j=7,8,9,10$, which are not uniform, generate large values of $\overline{\mathscr{E}}$. Note also that value of $E_{\widetilde{\omega}}^{*}$, computed with the identified building model corresponding to $\Pi_{7}^{2}$, is large. Moreover, it is not possible to identify the model of the structure through layouts $\Pi_{j}^{2}, j=8,9,10$.

Figures $12(\mathrm{a})$ and 12(b) present the displacements $\widehat{x}_{5}$ estimated by the proposed state observer using layouts 


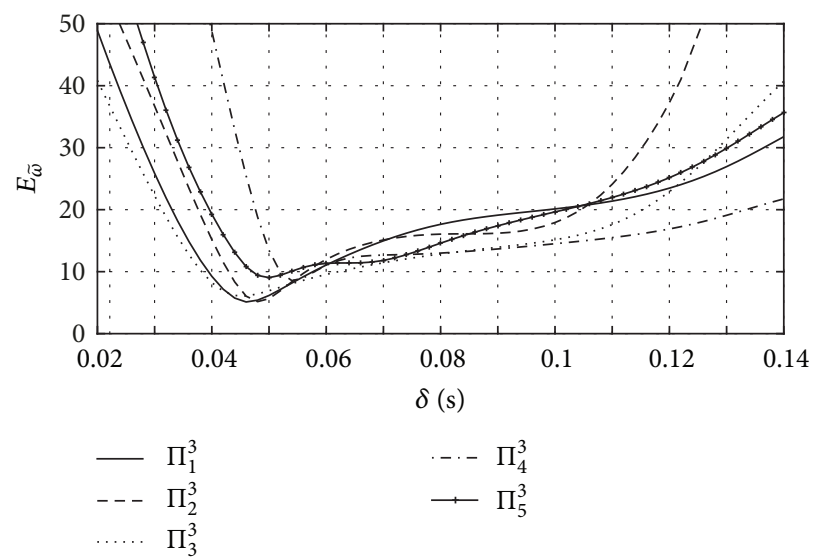

(a) $\Pi_{k}^{3}, k=1,2,3,4,5$

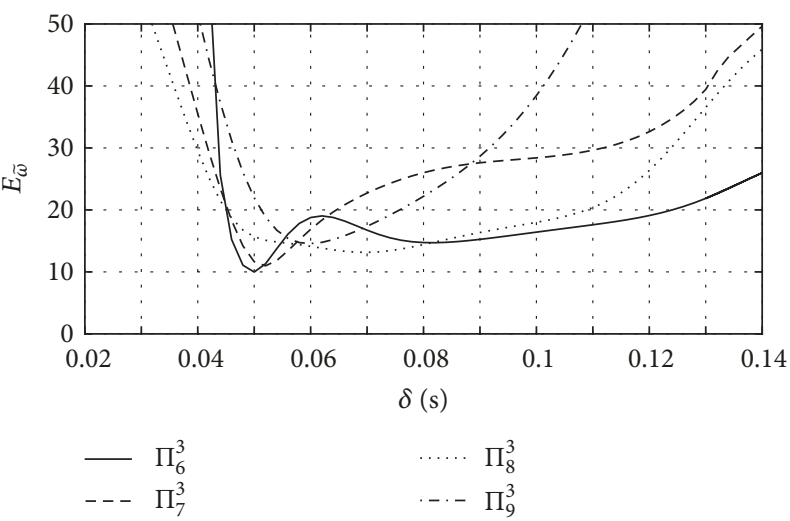

(b) $\Pi_{k}^{3}, k=6,7,8,9$

Figure 6: Function $E_{\widetilde{\omega}}(\delta)$ calculated with three instrumented floors.

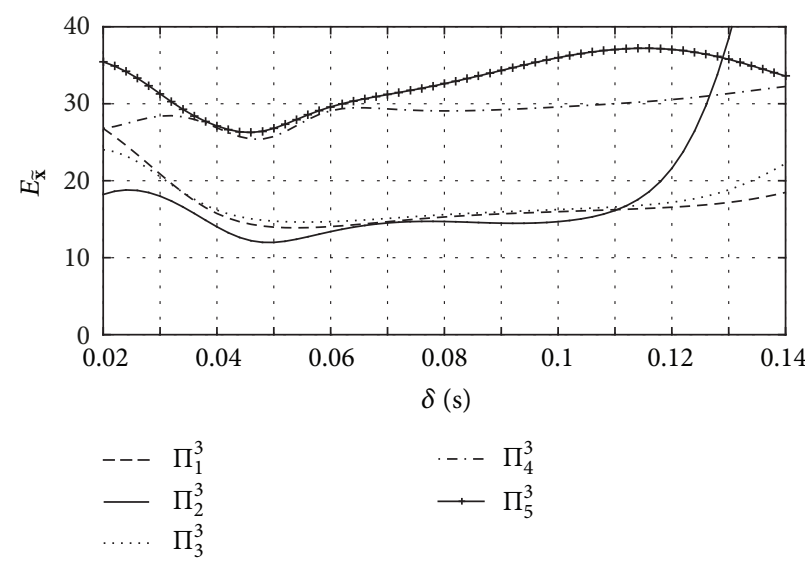

(a) $\Pi_{k}^{3}, k=1,2,3,4,5$

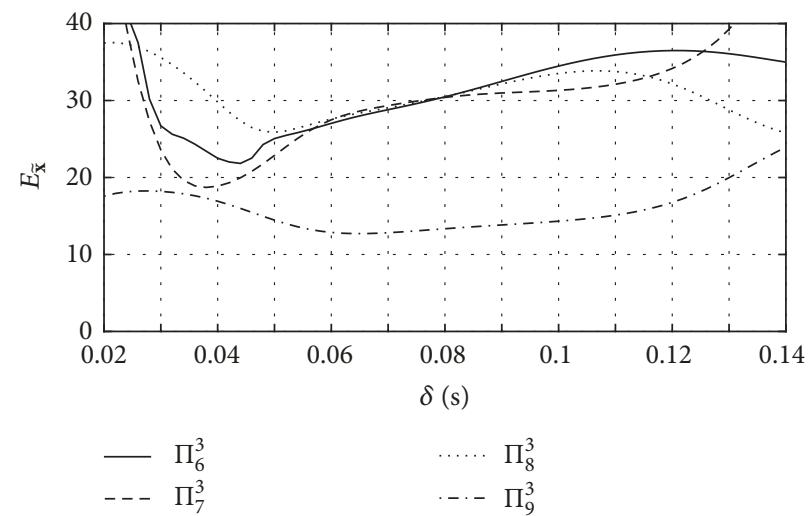

(b) $\Pi_{k}^{3}, k=6,7,8,9$

FIGURE 7: Function $E_{\widetilde{\mathbf{x}}}(\delta)$ computed with three instrumented floors.

$\Pi_{1}^{4}$ and $\Pi_{1}^{2}$, respectively. Distribution $\Pi_{1}^{4}$ was included for comparison purposes to show a degradation in the quality of the displacement reconstruction when using $\Pi_{1}^{2}$. In addition, Figure 13 compares the velocities $\hat{\dot{x}}_{5}$ obtained using these same distributions. Note that the quality of the reconstruction of $\hat{\dot{x}}_{5}$ is even worse than that of $\widehat{x}_{5}$ when using $\Pi_{1}^{2}$.

Note that in the distribution $\Pi_{1}^{4}$ only the acceleration $\ddot{x}_{3 a}$ is estimated by means of the cubic spline shape function, whereas in the distribution $\Pi_{1}^{2}$ three acceleration types $\ddot{x}_{1 a}$, $\ddot{x}_{3 a}$, and $\ddot{x}_{5 a}$ are reconstructed. Figures 14(a) and 14(b) depict the acceleration $\widehat{\ddot{x}}_{3 a}$ obtained with the distributions $\Pi_{1}^{4}$ and $\Pi_{1}^{2}$, respectively. It is shown that both estimates are close to the nominal acceleration $\ddot{x}_{3 a}$. On the other hand, Figure 15 presents the other two estimated acceleration types ${\tilde{\ddot{x}}_{1 a}}_{1 a}$ and $\widehat{\ddot{x}}_{5 a}$ corresponding to the distribution $\Pi_{1}^{2}$. By comparing Figures 14(b), 15(a), and 15(b), it is concluded that the acceleration that is best reconstructed in the distribution $\Pi_{1}^{2}$ is $\ddot{x}_{3 a}$.

7.5. Results with One Instrumented Floor. It is not possible to identify the model of the building with all distributions of one instrumented floor, since the reconstructed acceleration responses generate a regressor vector $\widehat{\phi}$, with which the covariance matrices $\mathbf{P}$ in (34) and (35) do not exist.

7.6. Parameter Estimates Obtained with Two, Three, Four, and Five Instrumented Floors. Define $\left(\Pi_{j}^{P}, \delta_{*}\right)$ as the $j$ th distribution of $P$ instrumented floors with its corresponding integration period $\delta^{*}$ of the LIF that produces $E_{\widetilde{\omega}}^{*}$. Table 6 presents the estimated parameters $\widehat{\theta}_{i}, i=1,2, \ldots, 9$, by the proposed technique with $\left(\Pi_{1}^{2}, \delta_{*}\right),\left(\Pi_{1}^{3}, \delta_{*}\right),\left(\Pi_{1}^{4}, \delta_{*}\right)$, and $\left(\Pi_{1}^{5}, \delta_{*}\right)$. Moreover, this Table shows parameters $\widehat{\omega}_{r}, \widehat{\zeta}_{r}, \widehat{m}_{r}$ and $\widehat{k}_{r}, r=1,2, \ldots, 5$ computed using those layouts. It is possible to observe that the error in all the estimated natural frequencies $\widehat{\omega}_{r}$ and damping factors $\hat{\zeta}_{r}$ is less than $4 \%$. Note that for $\left(\Pi_{1}^{5}, \delta_{*}\right),\left(\Pi_{1}^{4}, \delta_{*}\right),\left(\Pi_{1}^{3}, \delta_{*}\right)$, and $\left(\Pi_{1}^{2}, \delta_{*}\right)$, the error of the parameter estimates $\widehat{\theta}_{i}, i=1,2, \ldots, 9$, is less than $10 \%, 20 \%, 25 \%$, and $73 \%$, respectively. Moreover, for all these distributions, the maximum error obtained by estimating floor mases and stiffness parameters decreases as the number of instrumented floors increases. For example, 


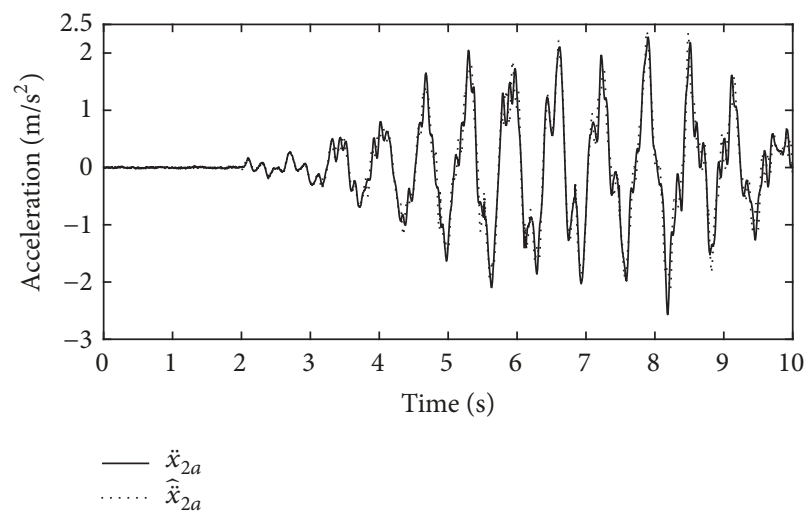

(a) Comparison between $\ddot{x}_{2 a}$ and $\widehat{\ddot{x}}_{2 a}$

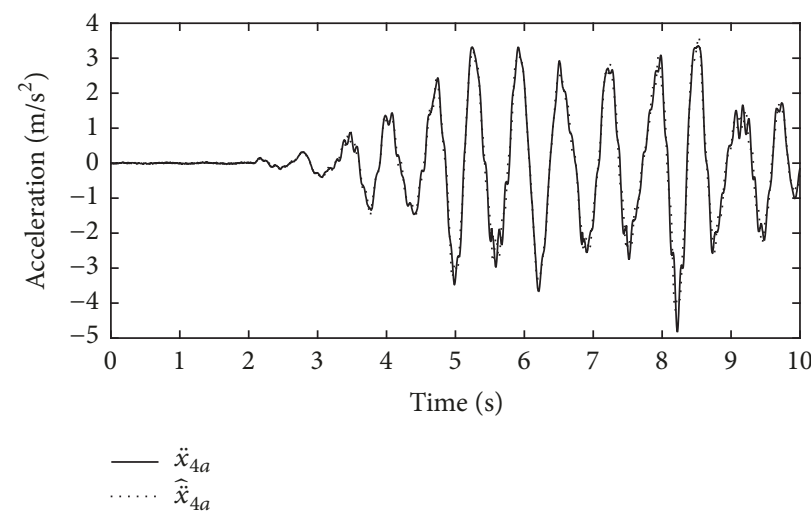

(b) Comparison between $\ddot{x}_{4 a}$ and $\widehat{\ddot{x}}_{4 a}$

FIGURE 8: Reconstructed signals $\widehat{\ddot{x}}_{2 a}$ and $\widehat{\ddot{x}}_{4 a}$ corresponding to the distribution $\Pi_{1}^{3}$.

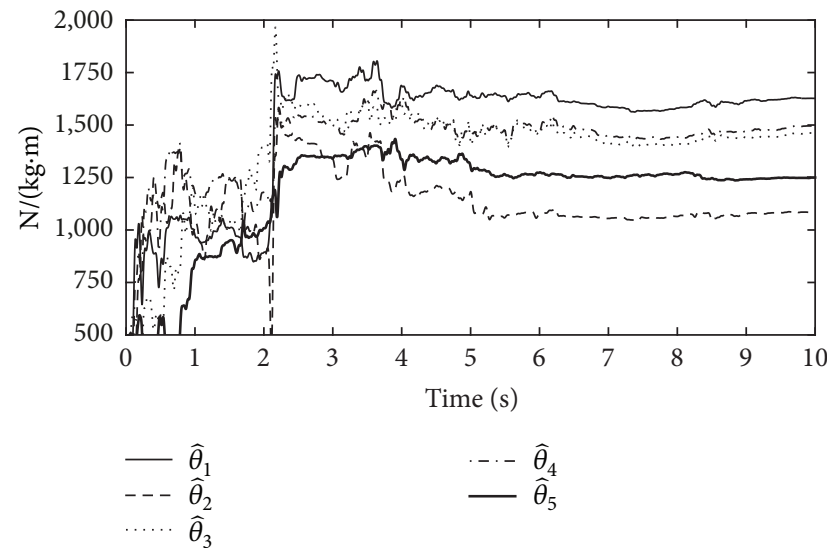

(a) $\widehat{\theta}_{i}, i=1,2,3,4,5$
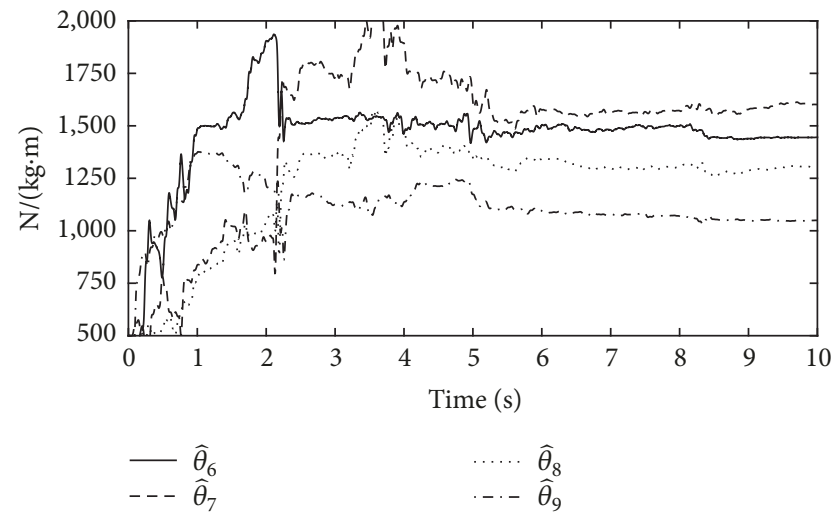

(b) $\widehat{\theta}_{i}, i=6,7,8,9$

FIGURE 9: Estimated vector $\widehat{\boldsymbol{\theta}}$ produced by the RLS and corresponding to the configuration $\Pi_{1}^{3}$.

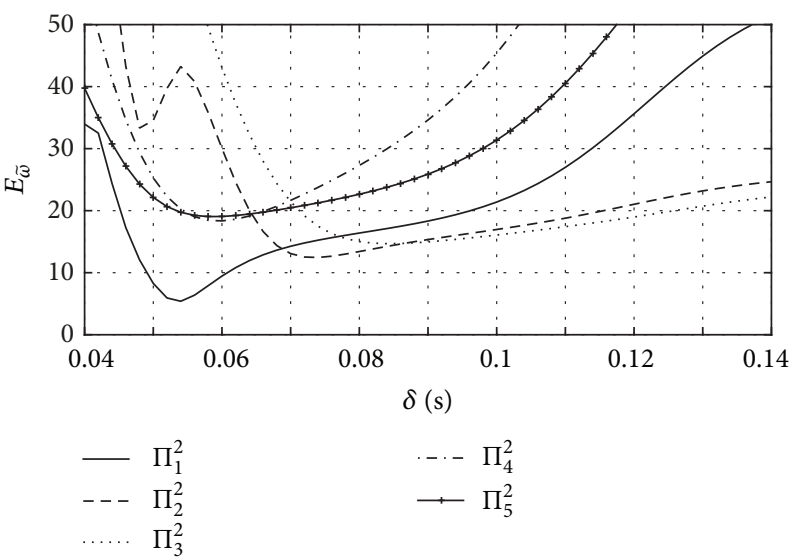

(a) $\Pi_{k}^{2}, k=1,2,3,4,5$

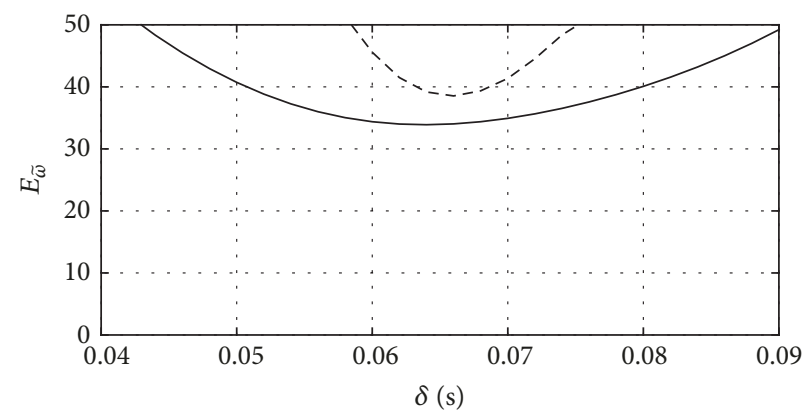

$\begin{array}{ll}- & \Pi_{6}^{2} \\ --- & \Pi_{7}^{2}\end{array}$

FIgURE 10: Function $E_{\widetilde{\omega}}(\delta)$ computed with two instrumented floors. 


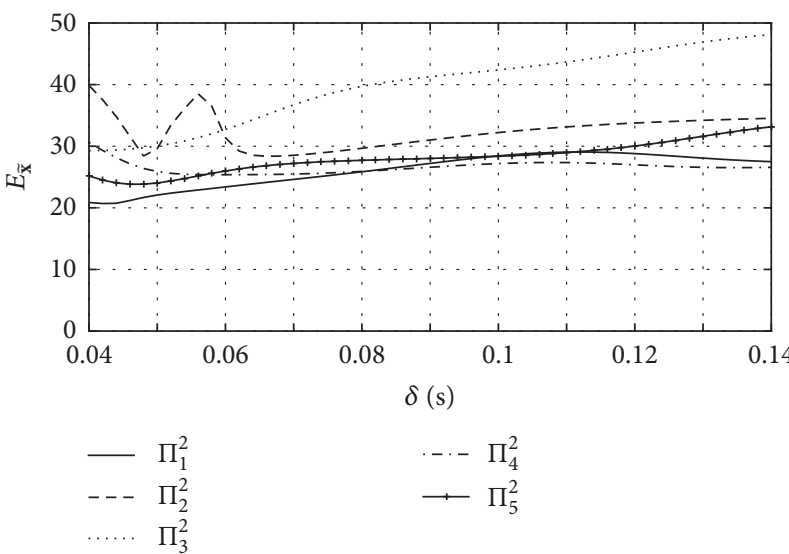

(a) $\Pi_{k}^{3}, k=1,2,3,4,5$

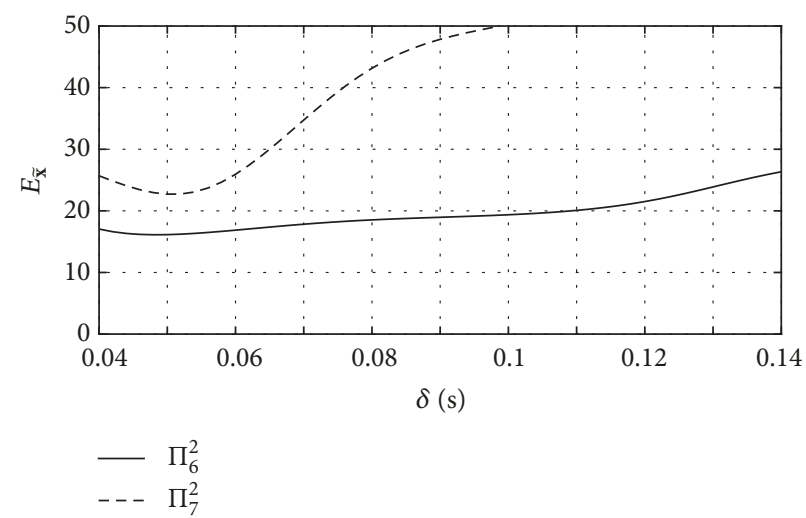

(b) $\Pi_{k}^{3}, k=6,7,8,9$

FiguRE 11: Function $E_{\tilde{\mathbf{x}}}(\delta)$ computed with two instrumented floors.

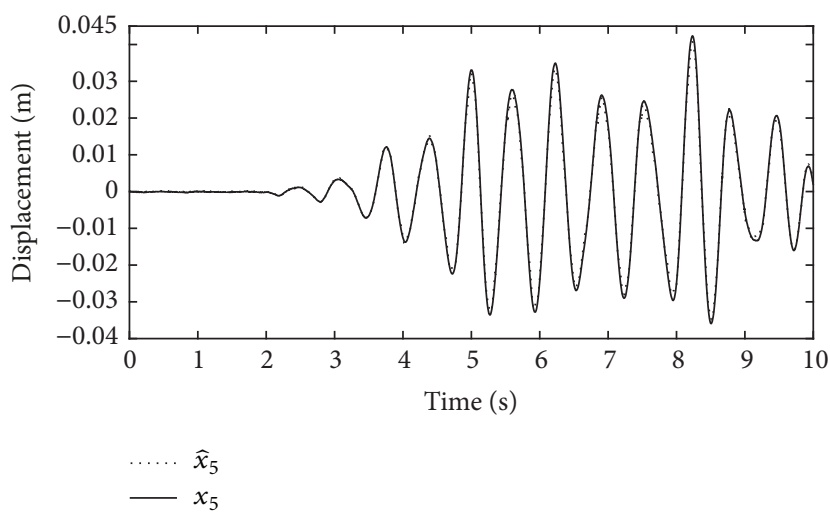

(a) State estimate $\widehat{x}_{5}$ corresponding to the distribution $\Pi_{1}^{4}$

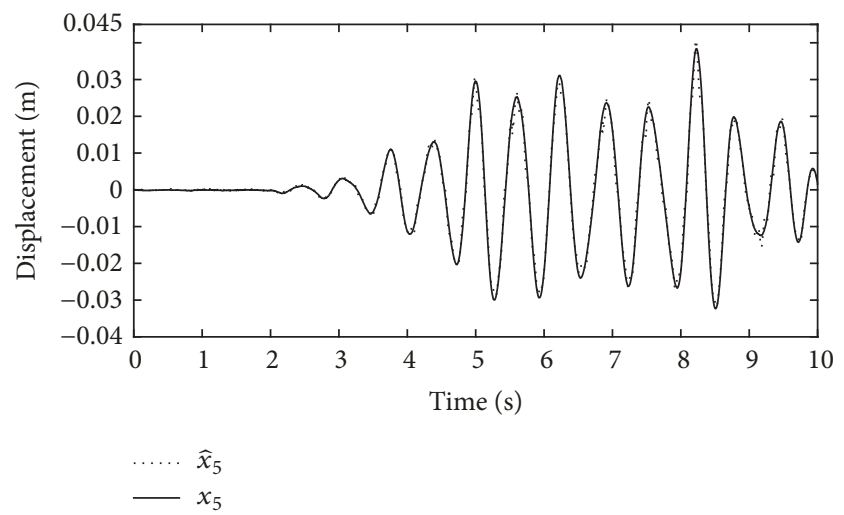

(b) State estimate $\widehat{x}_{5}$ corresponding to the distribution $\Pi_{1}^{2}$

FIGURE 12: Estimates produced by the state observer in the distributions $\Pi_{1}^{4}$ and $\Pi_{1}^{2}$.

the floor masses identified with layouts $\left(\Pi_{1}^{3}, \delta_{*}\right)$ and $\left(\Pi_{1}^{4}, \delta_{*}\right)$ have an error less than $18 \%$ and $8 \%$, respectively.

7.7. Summary of Experimental Results and Discussion. By comparing the results shown in Sections 7.1-7.6, the following points are concluded:

(i) The term $f^{*}=1 / \delta^{*}$ tends to decrease as the number of instrumented floors is reduced. Based on this fact and in our experience, the parameter $\delta$ should be selected so that $\omega_{c}=2 \pi / \delta$ takes a value within the interval $\omega_{c} \in\left[1.8 \omega_{\max } \sqrt{P / n}, 2.8 \omega_{\max } \sqrt{P / n}\right]$, where $\omega_{\max }$ is the maximum natural frequency of the structure.

(ii) Parameter $f^{\circ}$ does not depend on the number of instrumented floors. During experiments, term $f^{\circ}$ usually takes values close to $20 \mathrm{~Hz}$ with either complete or reduced instrumentation.

(iii) The quality of the estimated model mainly depends on the sensor location over the structure. When the building is instrumented at regular intervals over its height, then usually (1) the cubic spline shape function yields good results in the reconstruction of the unknown responses and (2) the parameter and state estimators have good performance.

(iv) In distributions of sensors that are not uniform along the building height, the cubic spline function produces responses with large errors, and therefore the quality of the estimated model is not guaranteed. In some cases, it is not even possible to identify the model and state of the structure using those responses.

(v) As expected, increasing the number of instrumented floors allows decreasing the error of the responses computed by the cubic spline function. This occurs with an increase in the cost of instrumentation. According to our experience, when the ratio $P / n$ is around $0.4-0.6$, good results in terms of the performance indexes $E_{\widetilde{\omega}}(\delta)$ and $E_{\tilde{\mathbf{x}}}(\delta)$ and the reproduction of displacements and velocities can be obtained. 


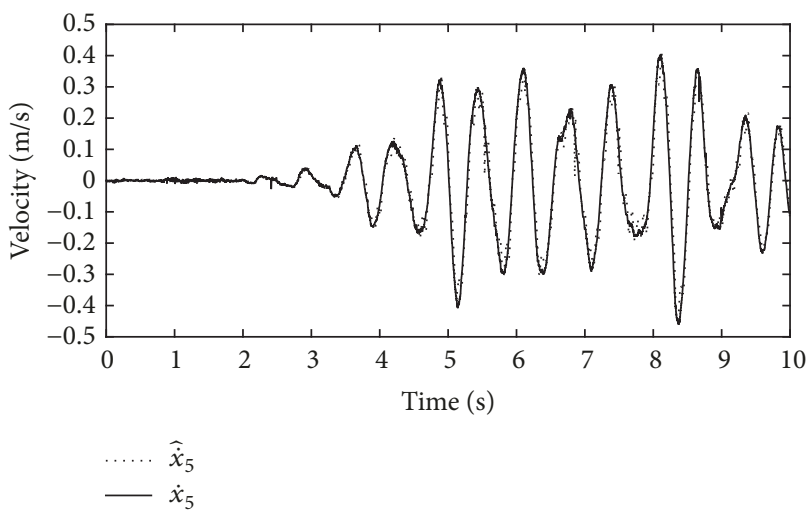

(a) State estimate $\hat{\dot{x}}_{5}$ corresponding to the distribution $\Pi_{1}^{4}$

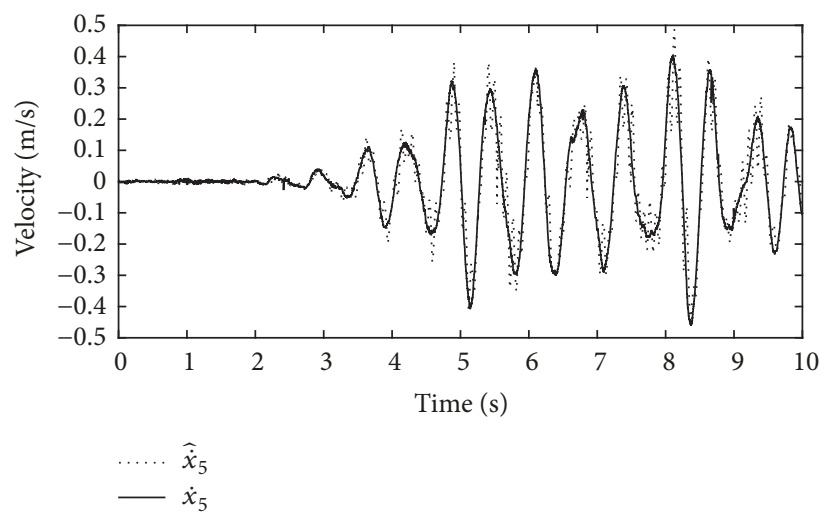

(b) State estimate $\hat{\dot{x}}_{5}$ corresponding to the distribution $\Pi_{1}^{2}$

FIGURE 13: Estimates produced by the state observer in the distributions $\Pi_{1}^{4}$ and $\Pi_{1}^{2}$.

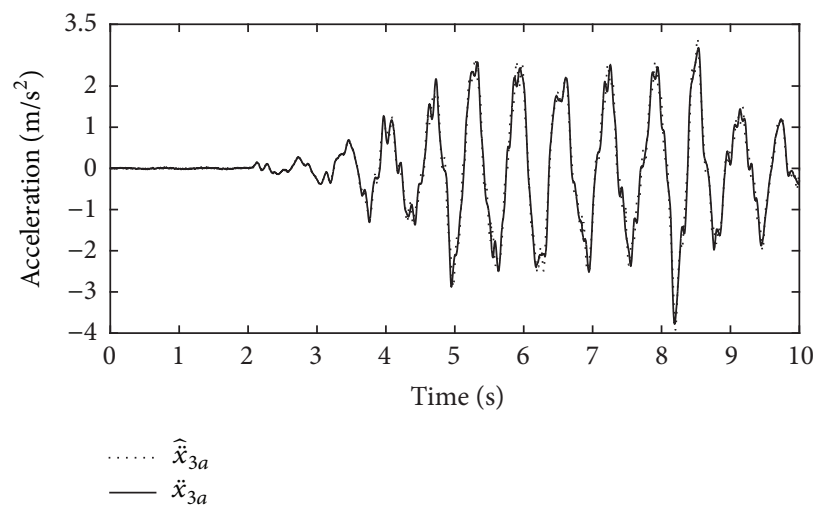

(a) Acceleration $\widehat{\ddot{x}}_{3 a}$ corresponding to the distribution $\Pi_{1}^{4}$
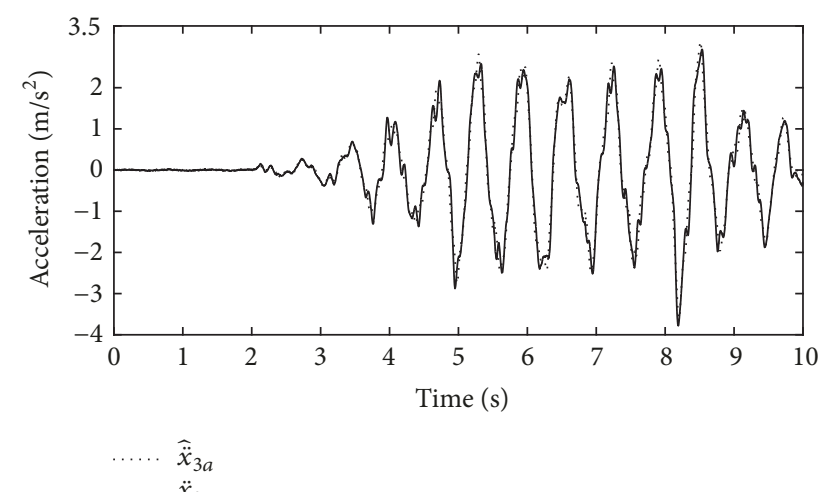

(b) Acceleration $\widehat{\ddot{x}}_{3 a}$ corresponding to the distribution $\Pi_{1}^{2}$

FIgURE 14: Acceleration $\widehat{\ddot{x}}_{3 a}$ obtained with the distributions $\Pi_{1}^{4}$ and $\Pi_{1}^{2}$.

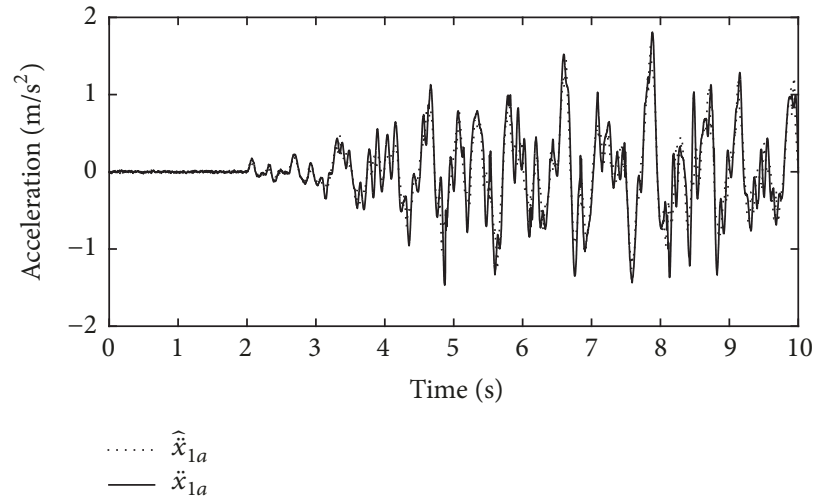

(a) Acceleration $\widehat{\ddot{x}}_{1 a}$ obtained with the distribution $\Pi_{1}^{2}$

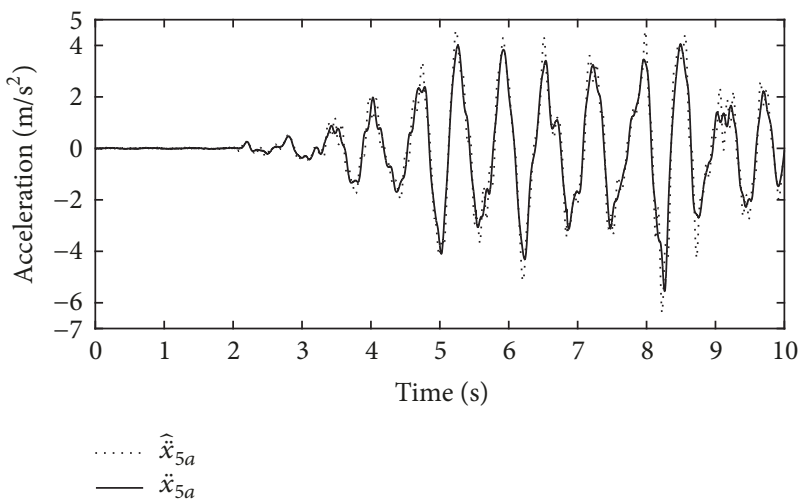

(b) Acceleration $\widehat{\ddot{x}}_{5 a}$ obtained with the distribution $\Pi_{1}^{2}$

FiguRE 15: Acceleration $\widehat{\ddot{x}}_{1 a}$ and acceleration $\widehat{\ddot{x}}_{5 a}$ corresponding to the distributions $\Pi_{1}^{4}$ and $\Pi_{1}^{2}$.

Values of $P / n$ smaller than 0.4 may yield numerical problems, as shown in the experimental results for the case of one instrumented floor.

Remark 5. The methods described in [1,3, 6-15] report good results in the parameter estimation of buildings with ratios
$P / n$ up to $0.5,0.33,0.5,0.43,0.5,0.33,0.25,0.5,0.18,0.5,0.5$, and 0.5 , respectively. Note that the ratio $P / n=0.5$ is the most common among them. Moreover, all of these methods operate offline with exception of the techniques presented in $[3,6]$, and most of them require transforming the building model from continuous one to its discrete time counterpart. 
TABLE 5: Results with two instrumented floors.

\begin{tabular}{lcccccccccc}
\hline $\begin{array}{l}\Pi_{j}^{2} \\
j\end{array}$ & Instrumented floors & Non-instrumented floors & $\overline{\mathscr{E}}$ & $E_{\tilde{\omega}}^{*}$ & $\delta^{*}[\mathrm{~s}]$ & $f_{c}^{*}[\mathrm{~Hz}]$ & $E_{\tilde{\mathbf{x}}}^{\circ}$ & $\delta^{\circ}[\mathrm{s}]$ & $f_{c}^{\circ}[\mathrm{Hz}]$ \\
\hline 1 & 024 & 135 & 1.01 & 5.40 & 0.054 & 18.52 & 20.7 & 0.042 & 23.81 \\
2 & 014 & 235 & 1.14 & 12.46 & 0.074 & 13.52 & 28.42 & 0.048 & 20.83 \\
3 & 015 & 234 & 1.23 & 14.68 & 0.084 & 11.90 & 29.29 & 0.04 & 25 \\
4 & 034 & 125 & 1.28 & 18.35 & 0.06 & 16.67 & 25.37 & 0.06 & 16.67 \\
5 & 025 & 134 & 0.99 & 19.05 & 0.058 & 17.24 & 23.83 & 0.046 & 21.74 \\
6 & 035 & 124 & 1.14 & 33.9 & 0.064 & 15.63 & 16.12 & 0.048 & 20.83 \\
7 & 045 & 123 & 1.47 & 38.54 & 0.06 & 16.67 & 22.71 & 0.05 & 20 \\
8 & 023 & 145 & 1.41 & - & - & - & - & - & - \\
9 & 013 & 245 & 1.39 & - & - & - & - & - & - \\
10 & 012 & 345 & 2.24 & - & - & - & - & - & - \\
\hline
\end{tabular}

TABLE 6: Parameter estimates corresponding to the distributions $\Pi_{1}^{2}, \Pi_{1}^{3}, \Pi_{1}^{4}$, and $\Pi_{1}^{5}$ of two, three, four, and five floors.

\begin{tabular}{|c|c|c|c|c|c|c|c|c|c|}
\hline $\begin{array}{l}\text { Nominal } \\
\text { value }\end{array}$ & Unit & $\begin{array}{c}\text { Estimate } \\
\left(\Pi_{1}^{5}, \delta^{*}\right)\end{array}$ & $\begin{array}{c}\text { Error } \\
\% \\
\end{array}$ & $\begin{array}{c}\text { Estimates } \\
\left(\Pi_{1}^{4}, \delta^{*}\right)\end{array}$ & $\begin{array}{c}\text { Error } \\
\% \\
\end{array}$ & $\begin{array}{c}\text { Estimates } \\
\left(\Pi_{1}^{3}, \delta^{*}\right)\end{array}$ & $\begin{array}{c}\text { Error } \\
\% \\
\end{array}$ & $\begin{array}{c}\text { Estimates } \\
\left(\Pi_{1}^{2}, \delta^{*}\right)\end{array}$ & $\begin{array}{c}\text { Error } \\
\% \\
\end{array}$ \\
\hline$\theta_{1}=1708.3$ & $1 / s^{2}$ & 1648.0 & 3.53 & 1629.0 & 4.64 & 1644.8 & 3.72 & 2940.5 & 72.13 \\
\hline$\theta_{2}=1144.3$ & $1 / s^{2}$ & 1134.4 & 0.86 & 1105.5 & 3.39 & 1121.8 & 1.96 & 1608.1 & 40.53 \\
\hline$\theta_{3}=1340.9$ & $1 / s^{2}$ & 1319.1 & 1.63 & 1262.9 & 5.82 & 1462.1 & 9.04 & 1104.4 & 17.64 \\
\hline$\theta_{4}=1340.9$ & $1 / s^{2}$ & 1337.7 & 0.24 & 1343.2 & 0.17 & 1504.3 & 12.19 & 1230.3 & 8.25 \\
\hline$\theta_{5}=1340.9$ & $1 / s^{2}$ & 1257.5 & 6.22 & 1526.8 & 13.87 & 1276.5 & 4.80 & 1150.7 & 14.19 \\
\hline$\theta_{6}=1340.9$ & $1 / s^{2}$ & 1307.8 & 2.47 & 1598.7 & 19.23 & 1466.1 & 9.33 & 1100.6 & 17.92 \\
\hline$\theta_{7}=1340.9$ & $1 / s^{2}$ & 1441.3 & 7.49 & 1336.6 & 0.32 & 1668.9 & 24.46 & 1392.7 & 3.86 \\
\hline$\theta_{8}=1.3409$ & $1 / s^{2}$ & 1456.6 & 8.63 & 1299.1 & 3.11 & 1344.9 & 0.30 & 924.4 & 31.06 \\
\hline$\theta_{9}=1340.9$ & $1 / s^{2}$ & 1346.1 & 0.38 & 1317.7 & 1.73 & 1065.0 & 20.57 & 1144.0 & 14.68 \\
\hline$\omega_{1}=11.07$ & $\mathrm{rad} / \mathrm{s}$ & 10.90 & 1.51 & 10.86 & 1.88 & 10.81 & 2.32 & 11.36 & 2.66 \\
\hline$\omega_{2}=31.80$ & $\mathrm{rad} / \mathrm{s}$ & 31.64 & 0.49 & 31.87 & 0.25 & 31.66 & 0.42 & 31.07 & 2.27 \\
\hline$\omega_{3}=48.86$ & $\mathrm{rad} / \mathrm{s}$ & 48.46 & 0.81 & 49.15 & 0.59 & 47.51 & 2.76 & 48.21 & 1.33 \\
\hline$\omega_{4}=61.63$ & $\mathrm{rad} / \mathrm{s}$ & 62.13 & 0.81 & 60.80 & 1.35 & 62.77 & 1.85 & 62.32 & 1.13 \\
\hline$\omega_{5}=70.14$ & $\mathrm{rad} / \mathrm{s}$ & 70.14 & 0 & 71.93 & 2.55 & 72.37 & 3.18 & 72.75 & 3.72 \\
\hline$\zeta_{1}=1$ & $\%$ & 1 & 0 & 1 & 0 & 1 & 0 & 0.99 & 1 \\
\hline$\zeta_{2}=1$ & $\%$ & 1 & 0 & 1 & 0 & 1 & 0 & 0.99 & 1 \\
\hline$\zeta_{3}=1.31$ & $\%$ & 1.3 & 0.76 & 1.31 & 0 & 1.28 & 2.29 & 1.3 & 0.76 \\
\hline$\zeta_{4}=1.57$ & $\%$ & 1.58 & 0.64 & 1.55 & 1.27 & 1.6 & 1.91 & 1.59 & 1.27 \\
\hline$\zeta_{5}=1.75$ & $\%$ & 1.75 & 0 & 1.79 & 2.29 & 1.8 & 2.86 & 1.81 & 3.43 \\
\hline$m_{1}=10.78$ & $\mathrm{Kg}$ & 10.78 & 0 & 10.78 & 0 & 10.78 & 0 & 10.78 & 0 \\
\hline$m_{2}=9.20$ & $\mathrm{Kg}$ & 9.27 & 0.77 & 9.44 & 2.57 & 8.27 & 10.09 & 15.70 & 70.62 \\
\hline$m_{3}=9.20$ & $\mathrm{Kg}$ & 9.86 & 7.20 & 8.30 & 9.77 & 9.75 & 5.95 & 16.78 & 82.43 \\
\hline$m_{4}=9.20$ & $\mathrm{Kg}$ & 8.95 & 2.73 & 9.93 & 7.93 & 8.56 & 6.92 & 13.26 & 44.16 \\
\hline$m_{5}=9.20$ & $\mathrm{Kg}$ & 9.68 & 5.26 & 9.79 & 6.42 & 10.81 & 17.53 & 10.72 & 16.48 \\
\hline$k_{1}=18415$ & $\mathrm{~N} / \mathrm{m}$ & 17766 & 3.53 & 17560 & 4.64 & 17731 & 3.72 & 31699 & 72.14 \\
\hline$k_{2}=12336$ & $\mathrm{~N} / \mathrm{m}$ & 12229 & 0.87 & 11917 & 3.39 & 12093 & 1.97 & 17335 & 40.53 \\
\hline$k_{3}=12336$ & $\mathrm{~N} / \mathrm{m}$ & 12402 & 0.87 & 12675 & 2.75 & 12443 & 0.87 & 19312 & 56.55 \\
\hline$k_{4}=12336$ & $\mathrm{~N} / \mathrm{m}$ & 12898 & 4.56 & 13272 & 7.57 & 14291 & 15.85 & 18471 & 49.73 \\
\hline$k_{5}=12336$ & $\mathrm{~N} / \mathrm{m}$ & 13035 & 5.66 & 12900 & 4.57 & 11516 & 6.65 & 12260 & 0.62 \\
\hline
\end{tabular}

Although the ratio $P / n=0.4$, corresponding to the proposed method, is larger than the ratios $P / n$ obtained with the offline techniques described in $[9,10,12]$, the proposed technique can be implemented online and can detect parameter changes produced by an anomaly in the structure due to a weak component or failure of elements. Moreover, unlike the methodology in [3], the proposed method does not need measurements of displacements and velocities of the floors, which are more difficult to obtain than floor acceleration measurements.

\section{Conclusions}

This paper presented a parameter estimation method and a high gain state observer that, respectively, identify the model and state of a shear building using acceleration 
measurements from only few floor levels and the responses at noninstrumented floors, which are computed through spline cubic shape functions. The building model, obtained by the LS parameter estimation method, is employed by the state observer. The performance of both the observer and the identification method was verified in a five-story experimental structure using one, two, three, four, and five instrumented floors. The experimental results give the following conclusions: (1) it is not possible to estimate the model and state of the structure with only one instrumented floor; (2) in general, the parameter and state estimators produce good results if the layout of recording sensors is uniform or approximately uniform along the height of the building; (3) increasing the number of instrumented floors improves the quality of the estimated model and reduces the state estimation error; (4) if the structure is not instrumented at regular or approximately regular intervals over its height, then a good quality of the identified model is not guaranteed. Even in this case, it may be not possible to identify the model and state of the structure; (5) a reasonable trade-off between estimators performance and the number of instrumented floors occurs when three floors are instrumented. It is worth mentioning that instrumenting two floors also yields good results as long as the distribution is regular.

\section{Appendix}

\section{A. Cubic Spline Shape Function Obtained Using the Response at $h=H$}

Let a building with height $H, n$ stories, be instrumented at its basement and at $P$ floors and be divided into $P$ subintervals, where each subinterval is delimited by two instrumented floors, as shown in Figure 1. The absolute acceleration at height $h$ within the $p$ th subinterval $\left[h_{p}, h_{p+1}\right]$, $p=0,1,2, \ldots, P-1$, is estimated through the following cubic shape spline function:

$$
\begin{aligned}
\widehat{\ddot{x}}_{a}(h)= & a_{p}+b_{p}\left(h-h_{p}\right)+d_{p}\left(h-h_{p}\right)^{2} \\
& +e_{p}\left(h-h_{p}\right)^{3}, \quad h \in\left[h_{p}, h_{p+1}\right]
\end{aligned}
$$

For the sake of simplicity the argument $t$ of signal $\widehat{\ddot{x}}_{a}(h, t)$ and parameters $a_{p}(t), b_{p}(t), c_{p}(t), a_{p}(t)$ has been omitted in this equation.

Coefficients $a_{p}, b_{p}, d_{p}$, and $e_{p}$ are computed by assuming that the $P$ cubic polynomials in (A.1) have continuous first and second derivatives with respect to the spatial variable $h$. This assumption allows obtaining the following equations [34]:

$$
\begin{aligned}
& \Delta h_{p}=h_{p+1}-h_{p}, \quad p=0,1,2, \ldots, P-1 \\
& a_{p}=r_{p}, \quad p=0,1,2, \ldots, P \\
& b_{p}=\frac{1}{\Delta h_{p}}\left(a_{p+1}-a_{p}\right)-\frac{\Delta h_{p}}{3}\left(2 d_{p}+d_{p+1}\right), \\
& \quad p=0,1,2, \ldots, P-1
\end{aligned}
$$

$$
\begin{aligned}
& e_{p}=\frac{d_{p+1}-d_{p}}{3 \Delta h_{p}}, \quad p=0,1,2, \ldots, P-1 \\
& \Delta h_{p-1} d_{p-1}+2\left(\Delta h_{p-1}+\Delta h_{p}\right) d_{p}+\Delta h_{p} d_{p+1} \\
& =\frac{3}{\Delta h_{p}}\left(r_{p+1}-r_{p}\right)-\frac{3}{\Delta h_{p-1}}\left(r_{p}-r_{p-1}\right), \\
& \quad p=1,2, \ldots, P-1
\end{aligned}
$$

First, the unknown parameters $\left\{d_{p}\right\}_{p=0}^{P}$ of the system of linear equations (A.6) are computed. Then, the constants $\left\{b_{p}\right\}_{p=0}^{P-1}$ and $\left\{e_{p}\right\}_{p=0}^{P-1}$ are obtained using equations (A.4) and (A.5), respectively. In order to solve the system of equations (A.6), two extra equations are needed, which are deduced from the boundary conditions $\ddot{x}_{a}^{\prime}(0)$ and $\ddot{x}_{a}^{\prime \prime}(H)$. Taking into account these conditions produces

$$
\begin{gathered}
\ddot{x}_{a}^{\prime}(0)=b_{0}, \\
\ddot{x}_{a}^{\prime \prime}(H)=2 d_{P}
\end{gathered}
$$

Substituting $\ddot{x}_{a}^{\prime}(0)=0$ and $\ddot{x}_{a}^{\prime \prime}(H)=0$, given in Table 1, into (A.7) yields

$$
\begin{array}{r}
b_{0}=0 \\
d_{P}=0
\end{array}
$$

Substituting $b_{0}=0$ into (A.4) and using (A.3) lead to

$$
\frac{1}{\Delta h_{0}}\left(r_{1}-r_{0}\right)-\frac{\Delta h_{0}}{3}\left(2 d_{0}+d_{1}\right)=0
$$

Expressions (A.6), (A.9), and (A.10) can be rewritten in matrix form as follows:

$$
\mathrm{SD}=\mathrm{V}
$$

S

$$
=\left[\begin{array}{cccccc}
2 \Delta h_{0} & \Delta h_{0} & 0 & 0 & \cdots & 0 \\
\Delta h_{0} & 2\left(\Delta h_{0}+\Delta h_{1}\right) & \Delta h_{1} & 0 & \cdots & 0 \\
0 & \Delta h_{1} & 2\left(\Delta h_{1}+\Delta h_{2}\right) & \Delta h_{2} & \cdots & 0 \\
\vdots & \vdots & \vdots & \ddots & \vdots & \\
0 & 0 & 0 & 0 & \cdots & 1
\end{array}\right]
$$




$$
\begin{aligned}
\mathbf{D} & =\left[\begin{array}{c}
d_{0} \\
d_{1} \\
d_{2} \\
\vdots \\
d_{P}
\end{array}\right], \\
\mathbf{V} & =\left[\begin{array}{c}
\frac{3}{\Delta h_{0}}\left(r_{1}-r_{0}\right) \\
\frac{3}{\Delta h_{1}}\left(r_{2}-r_{1}\right)-\frac{3}{\Delta h_{0}}\left(r_{1}-r_{0}\right) \\
\frac{3}{2}\left(r_{2}\right)-\frac{3}{\Delta h_{1}}\left(r_{2}-r_{1}\right) \\
\vdots \\
0
\end{array}\right]
\end{aligned}
$$

From (A.11), the matrix $\mathbf{D}$ is computed as follows:

$$
\mathbf{D}=\mathbf{S}^{-1} \mathbf{V} \text {, }
$$

where the inverse matrix $\mathbf{S}^{-1}$ exists, since $\mathbf{S}$ is a diagonally dominant matrix. Once matrix $\mathbf{D}$ is obtained, the parameters $\left\{b_{p}\right\}_{p=0}^{P-1}$ and $\left\{e_{p}\right\}_{p=0}^{P-1}$ are computed using (A.4) and (A.5).

\section{B. Cubic Spline Shape Function Deduced When the Response at $h=H$ Is Not Available}

In this case the building is divided into $P+1$ subintervals, as shown in Figure 1(b), where the subinterval $\Delta h_{P}$ is given by

$$
\Delta h_{P}=H-h_{P}
$$

The absolute acceleration at height $h$ within the $p$ th subinterval is computed with (A.1), where $p=0,1,2, \ldots, P$.

Since the cubic polynomials corresponding to the subintervals $\Delta h_{P-1}$ and $\Delta h_{P}$ have the same spatial derivative at $h=h_{P}$, the next equality is obtained [34]:

$$
\ddot{x}_{a}^{\prime}(H)=b_{P}=b_{P-1}+2 d_{P-1} \Delta h_{P-1}+3 e_{P-1} \Delta_{P-1}^{2}
$$

Moreover, the cubic spline function has the following second and third derivatives with respect to $h$ at the boundary $H$ :

$$
\begin{aligned}
& \ddot{x}_{a}^{\prime \prime}(H)=2 d_{P}+6 e_{P} \Delta_{P} \\
& \ddot{x}_{a}^{\prime \prime \prime}(H)=6 e_{P}
\end{aligned}
$$

Substituting the boundary conditions $\ddot{x}_{a}^{\prime \prime}(H)=\ddot{x}_{a}^{\prime \prime \prime}(H)=$ 0 , given in Table 1, into (B.3) and (B.4) yields

$$
\begin{aligned}
& d_{P}=0 \\
& e_{P}=0
\end{aligned}
$$

Moreover, the boundary condition $\ddot{x}_{a}^{\prime}(0)=0$ in Table 1 produces expression (A.10).

Coefficients $\left\{d_{p}\right\}_{p=0}^{P}$ are computed by solving the set of equations (A.6), (A.10), and (B.5). Subsequently, the parameters $\left\{b_{p}\right\}_{p=0}^{P}$ and $\left\{e_{p}\right\}_{p=0}^{P}$ are obtained using (A.4), (B.2) and (A.5), (B.6), respectively.

\section{Proof of Proposition 4}

Proof. Substituting the gain $\mathbf{L}=\left[\begin{array}{ll}\mathbf{O}_{\mathbf{n} \times \mathbf{n}} & -\gamma \mathbf{I}_{\mathbf{n} \times \mathbf{n}}\end{array}\right]^{T}$ into term $(\widehat{\mathbf{A}}-\mathbf{L} \widehat{\mathbf{D}})$ of $(47)$ produces

$$
\begin{aligned}
\dot{\tilde{\boldsymbol{\eta}}}(t) & =\mathbf{A}_{*} \widetilde{\boldsymbol{\eta}}(t)+\boldsymbol{\Xi}(t) \\
\mathbf{A}_{*} & =(\widehat{\mathbf{A}}-\mathbf{L} \widehat{\mathbf{D}}) \\
& =\left[\begin{array}{cc}
\mathbf{O}_{\mathbf{n} \times \mathbf{n}} & \mathbf{I}_{\mathbf{n} \times \mathbf{n}} \\
-(1+\gamma) \widehat{\mathbf{M}^{-1} \mathbf{K}} & -(1+\gamma) \widehat{\mathbf{M}^{-1} \mathbf{C}}
\end{array}\right],
\end{aligned}
$$

where matrices $\widehat{\mathbf{A}}$ and $\widehat{\mathbf{D}}$ are defined in (36) and (45), respectively.

Using expression $\widetilde{\boldsymbol{\eta}}(t)$ in (46) permits writing the homogeneous part of (C.1) as follows:

$$
\begin{aligned}
\widetilde{\mathbf{x}}(t) & +(1+\gamma) \widehat{\mathbf{M}^{-1} \mathbf{C}} \widetilde{\mathbf{x}}(t)+(1+\gamma) \widehat{\mathbf{M}^{-1} \mathbf{K}} \widetilde{\mathbf{x}}(t) \\
= & \mathbf{O}_{\mathbf{n} \times \mathbf{1}}
\end{aligned}
$$

Define the following equalities:

$$
\begin{aligned}
& \widehat{M^{-1} \mathbf{C}}=\widehat{M}^{-1} \widehat{C}, \\
& \widehat{M^{-1} \mathbf{K}}=\widehat{M}^{-1} \widehat{K}
\end{aligned}
$$

Using these definitions, it is possible to write (C.3) as

$$
\frac{1}{(1+\gamma)} \widehat{\mathbf{M}} \ddot{\mathbf{x}}(t)+\widehat{\mathbf{C}} \tilde{\mathbf{x}}(t)+\widehat{\mathbf{K}} \widetilde{\mathbf{x}}=\mathbf{O}_{\mathbf{n} \times \mathbf{1}}
$$

Modal analysis allows expressing (C.5) as the following $n$ uncoupled differential equations:

$$
\begin{aligned}
\frac{1}{(1+\gamma)} \widehat{m}_{i \bullet} \ddot{q}_{i}(t)+\widehat{c}_{i \bullet} \dot{q}_{i}(t)+\widehat{k}_{i \bullet} q_{i}(t)= & \\
& i=1,2, \ldots, n,
\end{aligned}
$$

where $q_{i}(t)$ denotes modal coordinate, and parameters $\widehat{m}_{i \bullet}$, $\widehat{c}_{i \bullet}$, and $\widehat{k}_{i \bullet}$ are estimates of the modal mass, damping, and stiffness, which are defined as

$$
\begin{aligned}
\widetilde{\mathbf{x}}(t) & =\sum_{r=1}^{n} \psi_{\mathbf{i}} q_{i}(t), \\
\widehat{m}_{i \bullet} & =\psi_{\mathbf{i}}^{T} \widehat{\mathbf{M}} \psi_{\mathbf{i}}, \\
\widehat{c}_{i \bullet} & =\psi_{\mathbf{i}}^{T} \widehat{\mathbf{C}} \psi_{\mathbf{i}}, \\
\widehat{k}_{i \bullet} & =\psi_{\mathbf{i}}^{T} \widehat{\mathbf{K}} \psi_{\mathbf{i}}
\end{aligned}
$$

where $i=1,2, \ldots, n$; variable $\psi_{\mathbf{i}}$ is the natural mode vector corresponding to the $i$ th natural frequency $\widehat{\omega}_{i}(40)$ of the estimated building model; i.e.,

$$
\left[\widehat{\mathbf{K}}-\widehat{\omega}_{i}^{2} \widehat{\mathbf{M}}\right] \psi_{\mathbf{i}}=\mathbf{O}_{\mathbf{n} \times \mathbf{1}}, \quad \widehat{\omega}_{i}=\sqrt{\frac{\widehat{k}_{i \bullet}}{\widehat{m}_{i \bullet}}}, i=1,2, \ldots, n
$$


Equation (C.6) is equivalent to the following expression:

$$
\begin{aligned}
& \ddot{q}_{i}+2(1+\gamma) \widehat{\zeta}_{i} \widehat{\omega}_{i} \dot{q}_{i}+(1+\gamma) \widehat{\omega}_{i}^{2} q_{i}=, \\
& \widehat{\zeta}_{i}=\frac{\widehat{c}_{i \bullet}}{\left(2 \widehat{m}_{i \bullet} \widehat{\omega}_{i}\right)},
\end{aligned}
$$

where $i=1,2, \ldots, n$ and $\widehat{\zeta}_{i}$ are the modal damping ratios of the identified building model.

Using equalities in (55), it is possible to write (C.9) as

$$
\ddot{q}_{i}+2 \zeta_{i}^{*} \omega_{i}^{*} \dot{q}_{i}+\omega_{i}^{* 2} q_{i}=0
$$

The characteristic equation corresponding to the $i$ th differential equation in (C.10) is given by

$$
s^{2}+2 \zeta_{i}^{*} \omega_{i}^{*} s+\omega_{i}^{* 2}=0, \quad i=1,2, \ldots, n
$$

The roots $\lambda_{i, 1}^{*}$ and $\lambda_{i, 2}^{*}, i=1,2, \ldots, n$, of (C.11) or equivalently the eigenvalues of $\mathbf{A}_{*}$ in (C.2), are given by expressions (53)-(55). Therefore, point (1)(a) of Proposition 4 has been proved.

On the other hand, substituting equalities in (55) into the characteristic equation (C.11) gives

$$
s^{2}+2(1+\gamma) \widehat{\zeta}_{i} \widehat{\omega}_{i} s+(1+\gamma) \widehat{\omega}_{i}^{2}=0, \quad, \quad i=1,2, \ldots, n
$$

Equation (C.12) can be rewritten as

$$
\begin{aligned}
1+\gamma \frac{\mathscr{Z}_{i}(s)}{\mathscr{Q}_{i}(s)} & =1+\gamma \mathscr{P}_{i}(s)=0, \\
\mathscr{P}_{i}(s) & =\frac{\mathscr{Z}_{i}(s)}{\mathscr{Q}_{i}(s)}=\frac{2 \widehat{\zeta}_{i} \widehat{\omega}_{i}\left(s+\widehat{\omega}_{i} / 2 \widehat{\zeta}_{i}\right)}{s^{2}+2 \widehat{\zeta}_{i} \widehat{\omega}_{i} s+\widehat{\omega}_{i}^{2}}
\end{aligned}
$$

According to the root locus technique, the two roots $\lambda_{i, 1}^{*}$ and $\lambda_{i, 2}^{*}$ in (53)-(55) of each characteristic equation in (C.13) traverse the path shown in Figure 16. It is possible to see that root locus has a break-in point where the two roots $\lambda_{i, 1}^{*}$ and $\lambda_{i, 2}^{*}$ return to the real axis. Let $\varrho_{i}$ be the value of the gain $\gamma$ in which the two roots $\lambda_{i, 1}^{*}$ and $\lambda_{i, 2}^{*}$ of the $i$ th characteristic equation become real. This gain $\varrho_{i}$ is computed by considering as zero the imaginary part of $\lambda_{i, 1}^{*}$ and $\lambda_{i, 2}^{*}$; i.e.,

$$
\begin{aligned}
\omega_{i}^{*} \sqrt{1-\zeta^{* 2}} & =0 \Longrightarrow \\
\zeta^{* 2} & =1 \Longrightarrow \\
(1+\gamma) \hat{\zeta}^{2} & =1 \Longrightarrow \\
\varrho_{i} & =\frac{1}{\widehat{\zeta}_{i}^{2}}-1
\end{aligned}
$$

Thus, increasing $\gamma$ from 0 to $\varrho_{i}$ allows moving the real part of the roots $\lambda_{i, 1}^{*}$ and $\lambda_{i, 2}^{*}$ further and further away from the left side of the real axis. Therefore, if $\gamma$ satisfies $0 \leq \gamma \leq \kappa=$ $\min \left[\varrho_{1}, \varrho_{2}, \ldots, \varrho_{n}\right]$, then the real part of the roots $\lambda_{i, 1}^{*}$ and $\lambda_{i, 2}^{*}$,

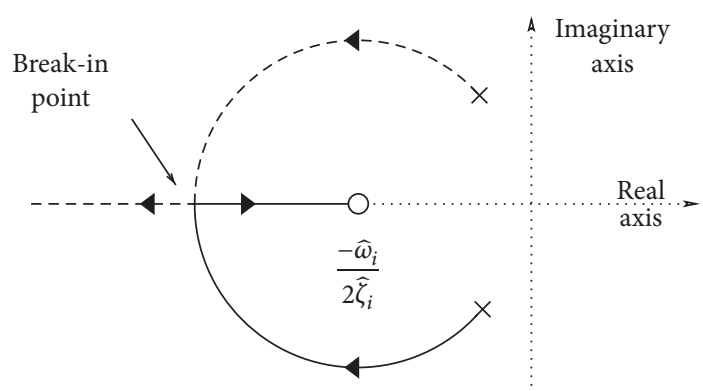

FIgURE 16: Root locus for the $i$ th characteristic equation in (C.13).

$i=1,2, \ldots, n$, becomes more and more negative as the gain $\gamma$ varies from zero to $\kappa$. Thus, point (1)(b) of Proposition 4 has been proved.

From Figure 16, it is possible to see that roots $\lambda_{i, 1}^{*}$ and $\lambda_{i, 2}^{*}$ of the $i$ th characteristic equation converge to the zeros of $\mathscr{Z}_{i}(s)$ as $\gamma \longrightarrow \infty$, which are given by

$$
\begin{aligned}
z_{i, 1} & =-\frac{\widehat{\omega}_{i}}{2 \widehat{\zeta}_{i}}, \\
z_{i, 2} & =-\infty, \\
& i=1,2, \ldots, n
\end{aligned}
$$

Therefore, point (2) of Proposition 4 has been proved.

In order to prove point (1)(c), the solution of (48) is used, which is given by

$$
\widehat{\boldsymbol{\eta}}(t)=\mathbf{e}^{\mathbf{A}_{*}} \hat{\boldsymbol{\eta}}(0)+\int_{0}^{t} \mathbf{e}^{\mathbf{A}_{*}(\mathbf{t}-\boldsymbol{\tau})} \boldsymbol{\Xi}(\tau) d \tau,
$$

where $\mathbf{e}^{\mathbf{A}_{*} \mathbf{t}}$ is the state transition matrix of system (48).

Since all the eigenvalues $\lambda_{i, 1}^{*}$ and $\lambda_{i, 2}^{*}, i=1,2, \ldots, n$, of $\mathbf{A}_{*}$ are different, the matrix $\mathbf{e}^{\mathbf{A}_{*} \mathbf{t}}$ can be rewritten as

$$
\mathrm{e}^{\mathbf{A}_{*} \mathbf{t}}=\mathfrak{M} \mathrm{e}^{\mathbf{D}_{*} \mathbf{t}} \mathbf{M}^{-1},
$$

where columns of matrix $\mathfrak{M}$ are the eigenvectors corresponding to the eigenvalues $\lambda_{i, 1}^{*}$ and $\lambda_{i, 2}^{*}, i=1,2, \ldots, n$; moreover, matrix $\mathbf{e}^{\mathbf{D}_{*} \mathbf{t}}$ is given by

$$
\begin{aligned}
& \mathbf{e}^{\mathbf{D}_{*} \mathbf{t}}=\left[\begin{array}{cccc}
e^{\lambda_{1,1}^{*} t} & 0 & \cdots & 0 \\
0 & e^{\lambda_{1,2}^{*} t} & \cdots & 0 \\
\vdots & \vdots & \ddots & \vdots \\
0 & 0 & \cdots & e^{\lambda_{n, 2}^{*} t}
\end{array}\right]=\mathbf{e}^{\mathbf{R}_{*} \mathbf{t} \mathbf{e}^{\mathcal{F}_{*} \mathbf{t}}} \\
& =\left[\begin{array}{cccc}
e^{-\sigma_{1}^{*} t} & 0 & \cdots & 0 \\
0 & e^{-\sigma_{1}^{*} t} & \cdots & 0 \\
\vdots & \vdots & \ddots & \vdots \\
0 & 0 & \cdots & e^{-\sigma_{n}^{*} t}
\end{array}\right]\left[\begin{array}{cccc}
e^{j \omega_{1}^{*} t} & 0 & \cdots & 0 \\
0 & e^{-j \omega_{1}^{*} t} & \cdots & 0 \\
\vdots & \vdots & \ddots & \vdots \\
0 & 0 & \cdots & e^{-j \omega_{n}^{*}}
\end{array}\right]
\end{aligned}
$$

Substituting the equality $\mathbf{e}^{\mathbf{D}_{*} \mathbf{t}}=\mathbf{e}^{\mathbf{R}_{*} \mathbf{t}} \mathbf{e}^{\mathscr{G}_{*} \mathbf{t}}$ (C.18) into (C.17) yields

$$
\mathbf{e}^{\mathbf{A}_{*} \mathbf{t}}=\mathbf{M e}^{\mathbf{R}_{*} \mathbf{t}} \mathbf{e}^{\mathcal{F}_{*} \mathbf{t}} \mathbf{M}^{-1}
$$


Matrix $\mathbf{e}^{\mathbf{A}_{*} \mathbf{t}}$ (C.20) satisfies

$$
\begin{array}{r}
\left\|\mathbf{e}^{\mathbf{A}_{*} \mathbf{t}}\right\|=\left\|\mathfrak{M} \mathbf{e}^{\mathbf{R}_{*} \mathbf{t}} \mathbf{e}^{\mathscr{T}_{*} \mathbf{t}} \mathbf{M}^{-\mathbf{1}}\right\| \leq \mu e^{-\sigma_{m}^{*} t}, \\
\sigma_{m}^{*}=\min _{1 \leq i \leq n}\left\{\sigma_{i}^{*}\right\}
\end{array}
$$$$
\mu=\|\mathfrak{M}\|\left\|\mathfrak{M}^{-1}\right\|,
$$$$
\left\|\mathbf{e}^{\mathbf{R}_{*} \mathbf{t}}\right\| \leq e^{-\sigma_{m}^{*} t},
$$$$
\left\|\mathbf{e}^{\mathcal{F}_{*}} \mathbf{t}\right\|=1
$$

where $\|\cdot\|=\|\cdot\|_{p}$ is the induced matrix norm, and $p$ can be 1,2 , or $\infty$.

The following inequality follows from (C.21):

$$
\left\|\mathbf{e}^{\mathbf{A}_{*} \mathbf{t}} \widehat{\boldsymbol{\eta}}(0)\right\| \leq \mu\|\widehat{\boldsymbol{\eta}}(0)\| e^{-\sigma_{m}^{*} t}
$$

Applying the matrix norm on both sides of (C.16) and using inequalities (C.21) and (C.23) produce

$$
\begin{aligned}
\|\widehat{\boldsymbol{\eta}}(t)\| \leq & e^{-(1+\gamma) \hat{\zeta}_{\bullet} \widehat{\omega}_{\bullet}} \mu\left[\|\widehat{\boldsymbol{\eta}}(0)\|-\frac{\|\boldsymbol{\Xi}(t)\|}{(1+\gamma) \widehat{\zeta}_{\bullet} \widehat{\omega}_{\bullet}}\right] \\
& +\frac{\mu\|\boldsymbol{\Xi}(t)\|}{(1+\gamma) \hat{\zeta}_{\bullet} \widehat{\omega}_{\bullet}} \\
\sigma_{m}^{*}= & (1+\gamma) \widehat{\zeta}_{\bullet} \widehat{\omega}_{\bullet}=(1+\gamma) \min _{1 \leq i \leq n}\left\{\widehat{\zeta}_{i} \widehat{\omega}_{i}\right\}
\end{aligned}
$$
to

Substituting the gain $\mathbf{L}(51)$ into $\boldsymbol{\Xi}(t)$ defined in (49) leads

$$
\begin{aligned}
\|\boldsymbol{\Xi}(t)\| \leq(1+\gamma)\left\|\left[\begin{array}{cc}
\mathbf{O}_{\mathbf{n} \times \mathbf{n}} & \frac{1}{1+\gamma} \mathbf{I}_{\mathbf{n} \times \mathbf{n}} \\
-\mathbf{M}^{-\mathbf{1}} \mathbf{K} & -\frac{\mathbf{M}^{-\mathbf{1}} \mathbf{C}}{n}
\end{array}\right]\right\|\|\boldsymbol{\eta}(t)\| \\
+\gamma\left\|\left[\mathbf{O}_{\mathbf{n} \times \mathbf{n}}-\mathbf{I}_{\mathbf{n} \times \mathbf{n}}\right]^{T}\right\|\left\|\boldsymbol{\varepsilon}_{\mathbf{a}}(t)\right\|, \\
\left\|\left[\mathbf{O}_{\mathbf{n} \times \mathbf{n}}-\mathbf{I}_{\mathbf{n} \times \mathbf{n}}\right]^{T}\right\|=1
\end{aligned}
$$

where $\overparen{\mathbf{M}^{-1} \mathbf{K}}$ and $\widetilde{\mathbf{M}^{-1} \mathbf{C}}$ are defined in (57).

Substituting inequality (C.25) into (C.24) and taking the limit of $\|\widehat{\boldsymbol{\eta}}(t)\|$ as $t \longrightarrow \infty$ yield (56). Thus, finally the point (1)(c) of Proposition 4 has been proved.

\section{Nomenclature}

LIF:

LS:

RLS:

$n \in \mathbb{N}$ :

$\mathbf{M} \in \mathbb{R}^{n x n}:$

$\mathbf{C} \in \mathbb{R}^{n x n}:$

$\mathbf{K} \in \mathbb{R}^{n x n}:$

$\mathbf{x}(t) \in \mathbb{R}^{n x 1}:$

$\dot{\mathbf{x}}(t) \in \mathbb{R}^{n x 1}:$

$\ddot{\mathbf{x}}(t) \in \mathbb{R}^{n x 1}:$

$\ddot{x}_{g}(t) \in \mathbb{R}:$

$\mathbf{l} \in \mathbb{R}^{n x 1}:$

$\ddot{\mathbf{x}}_{\mathbf{a}}(t) \in \mathbb{R}^{n x 1}$ :

$a_{0} \in \mathbb{R}:$

$a_{1} \in \mathbb{R}:$

$\omega_{i} \in \mathbb{R}^{+}$:

$\zeta_{i} \in \mathbb{R}^{+}:$

$\mathbf{A} \in \mathbb{R}^{2 n \times 2 n}$ :

$\mathbf{B} \in \mathbb{R}^{2 n x 1}:$

$\mathbf{D} \in \mathbb{R}^{n \times 2 n}:$

$\boldsymbol{\eta}(t) \in \mathbb{R}^{2 n x 1}:$

$P \in \mathbb{N}$ :

$\Gamma \in \mathbb{R}^{P x n}:$

$\ddot{\mathbf{u}}(t) \in \mathbb{R}^{n x(2 n-1)}$ :

$\boldsymbol{\theta} \in \mathbb{R}^{(2 n-1) x 1}:$

?:

$I_{\alpha}\{\rho(t)\}:$

$\delta \in \mathbb{R}^{+}:$

$T_{s} \in \mathbb{R}^{+}:$

$\mathscr{L}[\cdot]:$

$\phi(t) \in \mathbb{R}^{n x(2 n-1)}$;

$\chi(t) \in \mathbb{R}^{n x 1}$ :

$G_{\alpha}(s):$

$\omega_{c} \in \mathbb{R}^{+}$:

$f_{c} \in \mathbb{R}^{+}:$

$\mathscr{H}_{i}(s), i=1,2,3$ : Band pass filters

Least Squares

$\mathrm{N} \cdot \mathrm{s} / \mathrm{m}$ $\mathrm{m} / \mathrm{s}^{2}$ earthquake, $\mathrm{m} / \mathrm{s}^{2}$

Vector of ones floors, $\mathrm{m} / \mathrm{s}^{2}$ $\mathrm{rad} / \mathrm{s}$ building building excited building basement, $\mathrm{m} / \mathrm{s}^{2}$ $\mathrm{N} /(\mathrm{kg} \cdot \mathrm{m})$ the LIF, $s$

Laplace operator

Regressor vector

model

$\alpha$ th-order LIF $\mathrm{rad} / \mathrm{s}$ $\mathrm{Hz}$
Linear Integral Filter

Recursive Least Squares

Number of floors of a building

Mass matrix of the building, $\mathrm{kg}$

Damping matrix of the building,

Stiffness matrix of the building, $\mathrm{N} / \mathrm{m}$

Displacement vector of the floors relative to the building basement, $\mathrm{m}$

Velocity vector of the floors relative

to the building basement, $\mathrm{m} / \mathrm{s}$

Acceleration vector of the floors relative to the building basement,

Ground acceleration produced by an

Absolute acceleration vector of the

Rayleigh damping coefficient, 1/s

Rayleigh damping coefficient, $s$

Natural frequency of the $i$ th mode,

Damping ratio of the $i$ th mode, $\%$

State matrix of a seismically excited

Input vector of a seismically excited

Output matrix of a seismically

State of a seismically excited building Number of instrumented floors

Localization matrix of accelerometers

Matrix composed of acceleration of floors relative to the building

Stiffness/mass vector to be identified,

Superscript used in estimated parameters or signals

\section{LIF representing $\alpha$ integrals over} finite time intervals of $\rho(t)$

Time integration window length of

Sampling period, $s$

Output of the parameterization

Cut-off frequency of the filter $G_{\alpha}(s)$,

Cut-off frequency of the filter $G_{\alpha}(s)$, 


\begin{tabular}{|c|c|}
\hline$H \in \mathbb{R}^{+}:$ & Building height \\
\hline$h_{p} \in \mathbb{R}^{+}:$ & Height of the $p$ th instrumented floor \\
\hline$r_{p} \in \mathbb{R}:$ & $\begin{array}{l}\text { Absolute acceleration of the } p \text { th } \\
\text { instrumented floor, } \mathrm{m} / \mathrm{s}^{2}\end{array}$ \\
\hline$\ddot{x}(h, t) \in \mathbb{R}:$ & $\begin{array}{l}\text { Absolute acceleration at height } h \text { of } \\
\text { the building, } \mathrm{m} / \mathrm{s}^{2}\end{array}$ \\
\hline$\ddot{x}_{w a}(t) \in \mathbb{R}:$ & $\begin{array}{l}\text { Absolute acceleration at the } w \text { th } \\
\text { noninstrumented floor, } \mathrm{m} / \mathrm{s}^{2}\end{array}$ \\
\hline$h_{*} \in \mathbb{R}^{+}:$ & $\begin{array}{l}\text { Height at the } w \text { th noninstrumented } \\
\text { floor }\end{array}$ \\
\hline$\Delta_{p} \in \mathbb{R}^{+}:$ & $\begin{array}{l}\text { Subinterval delimited by two } \\
\text { instrumented floors }\end{array}$ \\
\hline$a_{p}, b_{p}, d_{p}, e_{p}:$ & $\begin{array}{l}\text { Coefficients of the } p \text { th cubic } \\
\text { polynomial }\end{array}$ \\
\hline $\boldsymbol{\epsilon}(t) \in \mathbb{R}^{n x 1}:$ & $\begin{array}{l}\text { Error between estimated and } \\
\text { nominal relative acceleration, } \mathrm{m} / \mathrm{s}^{2}\end{array}$ \\
\hline $\boldsymbol{\epsilon}_{\mathbf{a}}(t) \in \mathbb{R}^{n x 1}:$ & $\begin{array}{l}\text { Error between estimated and } \\
\text { nominal absolute acceleration, } \mathrm{m} / \mathrm{s}^{2}\end{array}$ \\
\hline $\boldsymbol{\epsilon}_{\mathbf{u}}(t) \in \mathbb{R}^{n x(2 n-1)}:$ & $\begin{array}{l}\text { Matrix composed of errors between } \\
\text { estimated and nominal relative } \\
\text { acceleration, } \mathrm{m} / \mathrm{s}^{2}\end{array}$ \\
\hline$\lambda(t) \in \mathbb{R}^{n x 1}:$ & $\begin{array}{l}\text { Signal depending on the acceleration } \\
\text { estimation errors }\end{array}$ \\
\hline$N \in \mathbb{N}:$ & Number of samples used by the LS \\
\hline $\mathbf{P} \in \mathbb{R}^{(2 n-1) x(2 n-1)}:$ & Covariance matrix of the LS or RLS \\
\hline $\mathbf{L} \in \mathbb{R}^{n \times 2 n}:$ & State observer gain matrix \\
\hline$\gamma \in \mathbb{R}^{+}:$ & $\begin{array}{l}\text { Multiplying factor of the state } \\
\text { observer gain matrix }\end{array}$ \\
\hline$\Pi_{j}^{P}:$ & $\begin{array}{l}j \text { th distribution of } P \text { instrumented } \\
\text { floors }\end{array}$ \\
\hline $\mathscr{E}_{w} \in \mathbb{R}^{+}:$ & $\begin{array}{l}\text { Metric to determine the effectiveness } \\
\text { in the reconstruction of the } w \text { th } \\
\text { noninstrumented floor }\end{array}$ \\
\hline$\overline{\mathscr{E}} \in \mathbb{R}^{+}:$ & $\begin{array}{l}\text { Mean value of a set of errors } \mathscr{E}_{w} \text { of a } \\
\text { distribution of instrumented floors }\end{array}$ \\
\hline$\tilde{\omega}_{i} \in \mathbb{R}^{+}:$ & $\begin{array}{l}\text { Identification error in percentage of } \\
\text { the } i \text { th estimated natural frequency, } \\
\%\end{array}$ \\
\hline$E_{\widetilde{\omega}}(\delta) \in \mathbb{R}^{+}:$ & $\begin{array}{l}\text { Performance index that measures the } \\
\text { quality of the identified model }\end{array}$ \\
\hline$E_{\widetilde{\omega}}^{*} \in \mathbb{R}^{+}:$ & Minimum value of $E_{\widetilde{\omega}}(\delta)$ \\
\hline$\delta^{*} \in \mathbb{R}^{+}:$ & $\begin{array}{l}\text { Time integration window length that } \\
\text { produces the minimum value of } \\
E_{\widetilde{\omega}}(\delta), \mathrm{s}\end{array}$ \\
\hline & The reciprocal of $\delta^{*}, \mathrm{~Hz}$ \\
\hline$E_{\widetilde{\mathbf{x}}}(\delta) \in \mathbb{R}^{+}:$ & $\begin{array}{l}\text { Performance of the high gain state } \\
\text { observer }\end{array}$ \\
\hline & Minimum value of function $E_{\widetilde{\mathbf{x}}}(\delta)$ \\
\hline$\delta^{\circ} \in \mathbb{R}^{+}:$ & $\begin{array}{l}\text { Time integration window length that } \\
\text { produces the minimum value of } \\
E_{\widetilde{\mathbf{x}}}(\delta), \mathrm{s}\end{array}$ \\
\hline $3^{+}:$ & The reciprocal of $\delta^{\circ}, \mathrm{Hz}$. \\
\hline
\end{tabular}

\section{Data Availability}

The data used to support the findings of this study are available from the corresponding author upon request.

\section{Conflicts of Interest}

The authors declare that there are no conflicts of interest regarding the publication of this paper.

\section{Acknowledgments}

Antonio Concha acknowledges Programa para el Desarrollo Profesional Docente (PRODEP-SEP) for supporting this research. Luis Alvarez-Icaza acknowledges support from UNAM-PAPIIT Grant IN104218.

\section{References}

[1] F. Amini and Y. Hedayati, "Underdetermined blind modal identification of structures by earthquake and ambient vibration measurements via sparse component analysis," Journal of Sound and Vibration, vol. 366, pp. 117-132, 2016.

[2] M. Hoshiya and E. Saito, "Structural identification by extended Kalman filter," Journal of Engineering Mechanics, vol. 110, no. 12, pp. 1757-1770, 1984.

[3] C. G. Koh, L. M. See, and T. Balendra, "Estimation of structural parameters in time domain. A substructure approach," Earthquake Engineering \& Structural Dynamics, vol. 20, no. 8, pp. 787-801, 1991.

[4] A. Corigliano and S. Mariani, "Parameter identification in explicit structural dynamics: Performance of the extended Kalman filter," Computer Methods Applied Mechanics and Engineering, vol. 193, no. 36-38, pp. 3807-3835, 2004.

[5] S. J. Ghosh, D. Roy, and C. S. Manohar, "New forms of extended Kalman filter via transversal linearization and applications to structural system identification," Computer Methods Applied Mechanics and Engineering, vol. 196, no. 49-52, pp. 5063-5083, 2007.

[6] J. Zhou, A. Mita, and L. Mei, "An improved bayesian structural identification using the first two derivatives of log-likelihood measure," Journal of Structures, vol. 2015, pp. 1-9, 2015.

[7] S. Mukhopadhyay, H. Lus, and R. Betti, "Structural identification with incomplete instrumentation and global identifiability requirements under base excitation," Structural Control and Health Monitoring, vol. 22, no. 7, pp. 1024-1047, 2015.

[8] P. Yuan, Z. Wu, and X. Ma, "Estimated mass and stiffness matrices of shear building from modal test data," Earthquake Engineering \& Structural Dynamics, vol. 27, no. 5, pp. 415-421, 1998.

[9] J.-N. Juang, M. Phan, L. G. Horta, and R. W. Longman, "Identification of observer/Kalman filter Markov parameters: theory and experiments," Journal of Guidance, Control, and Dynamics, vol. 16, no. 2, pp. 320-329, 1993.

[10] H. Lus, R. Betti, and R. W. Longman, "Identification of linear structural systems using earthquake-induced vibration data," Earthquake Engineering \& Structural Dynamics, vol. 28, no. 11, pp. 1449-1467, 1999.

[11] F. Vicario, M. Q. Phan, R. Betti, and R. W. Longman, "Outputonly observer/Kalman filter identification (O $\left.{ }^{3} \mathrm{KID}\right)$," Structural Control and Health Monitoring, vol. 22, no. 5, pp. 847-872, 2015.

[12] Y. Kaya, S. Kocakaplan, and E. Şafak, "System identification and model calibration of multi-story buildings through estimation of vibration time histories at non-instrumented floors," Bulletin of Earthquake Engineering, vol. 13, no. 11, pp. 3301-3323, 2015. 
[13] G. Hegde and R. Sinha, "Method of modal identification of torsionally-coupled buildings using earthquake responses," in Proceedings of the The 14th World Conference on Earthquake Engineering, pp. 1-8, 2008.

[14] C. S. Huang, "Structural identification from ambient vibration measurement using the multivariate AR model," Journal of Sound and Vibration, vol. 241, no. 3, pp. 337-359, 2001.

[15] S. Chakraverty, "Identification of structural parameters of multistorey shear buildings from modal data," Earthquake Engineering \& Structural Dynamics, vol. 34, no. 6, pp. 543-554, 2005.

[16] H. Garnier and L. Wang, Identification of Continuous-time Models from Sampled Data, Springer-Verlag, London, UK, 2008.

[17] R. Garrido and A. Concha, "Estimation of the parameters of structures using acceleration measurements," in Proceedings of the Universite Libre de Bruxelles, pp. 1791-1796, Belgium, July 2012.

[18] M. P. Limongelli, "Optimal location of sensors for reconstruction of seismic responses through spline function interpolation," Earthquake Engineering \& Structural Dynamics, vol. 32, no. 7, pp. 1055-1074, 2003.

[19] M. P. Limongelli, "Performance evaluation of instrumented buildings," ISET Journal of Earthquake Technology, vol. 42, no. 2-3, pp. 47-61, 2005.

[20] F. E. Udwadia, "Methodology for optimum sensor locations for parameter identification in dynamic systems," Journal of Engineering Mechanics, vol. 120, no. 2, pp. 368-390, 1994.

[21] E. Heredia-Zavoni and L. Esteva, "Optimal instrumentation of uncertain structural systems subject to earthquake ground motions," Earthquake Engineering \& Structural Dynamics, vol. 27, no. 4, pp. 343-362, 1998.

[22] M. M. Abdullah, A. Richardson, and J. Hanif, "Placement of sensors/actuators on civil structures using genetic algorithms," Earthquake Engineering \& Structural Dynamics, vol. 30, no. 8, pp. 1167-1184, 2001.

[23] J. Li and S. S. Law, "Substructural response reconstruction in wavelet domain," Journal of Applied Mechanics, vol. 78, no. 4, Article ID 041010, 10 pages, 2011.

[24] S. S. Law, J. Li, and Y. Ding, "Structural response reconstruction with transmissibility concept in frequency domain," Mechanical Systems and Signal Processing, vol. 25, no. 3, pp. 952-968, 2011.

[25] J. He, X. Guan, and Y. Liu, "Structural response reconstruction based on empirical mode decomposition in time domain," Mechanical Systems and Signal Processing, vol. 28, pp. 348-366, 2012.

[26] Z. Wan, S. Li, Q. Huang, and T. Wang, "Structural response reconstruction based on the modal superposition method in the presence of closely spaced modes," Mechanical Systems and Signal Processing, vol. 42, no. 1-2, pp. 14-30, 2014.

[27] A. K. Chopra, Dynamics of Structures: Theory and Applications to Earthquake Engineering, Prentice Hall, Englewood Cliffs, NJ, USA, 2001.

[28] G. A. Papagiannopoulos and G. D. Hatzigeorgiou, "On the use of the half-power bandwidth method to estimate damping in building structures," Soil Dynamics and Earthquake Engineering, vol. 31, no. 7, pp. 1075-1079, 2011.

[29] J. Butterworth, J. H. Lee, and B. Davidson, "Experimental determination of modal damping from full scale testing," in Proceedings of the 13th world conference on earthquake engineering, vol. 310, Vancouver, Canada, 2004.
[30] A. Devin and P. Fanning, "Impact of nonstructural components on modal response and structural damping," in Topics on the Dynamics of Civil Structures, vol. 1, pp. 415-421, Springer, New York, NY, USA, 2012.

[31] T. Söderström and P. Stoica, System Identification, Prentice Hall, New York, NY, USA, 1989.

[32] P. Ioannou and B. Fidan, Adaptive Control Tutorial, vol. 11, SIAM, Philadelphia, USA, 2006.

[33] R. K. Goel, "Mode-based procedure to interpolate strong motion records of instrumented buildings," ISET Journal of Earthquake Technology, vol. 45, no. 3-4, pp. 97-113, 2008.

[34] R. L. Burden and J. D. Faires, "Análisis numérico," Thomson Learning, 2002. 


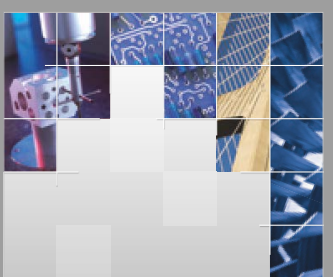

\section{Enfincering}
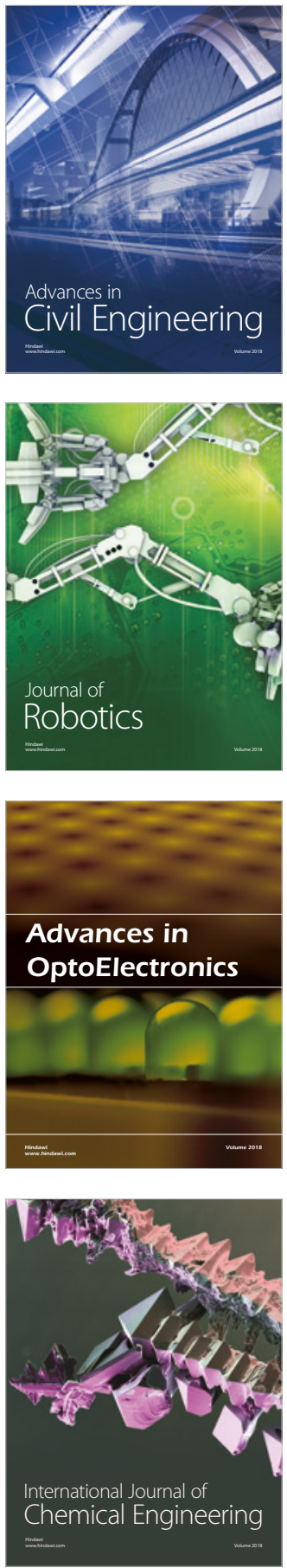

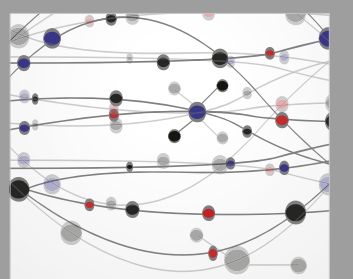

\section{Rotating \\ Machinery}

The Scientific World Journal

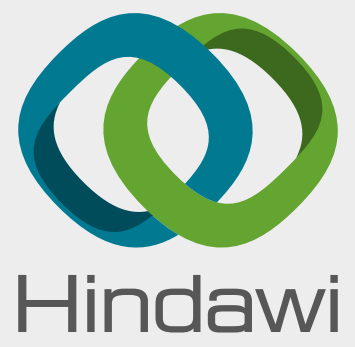

Submit your manuscripts at

www.hindawi.com
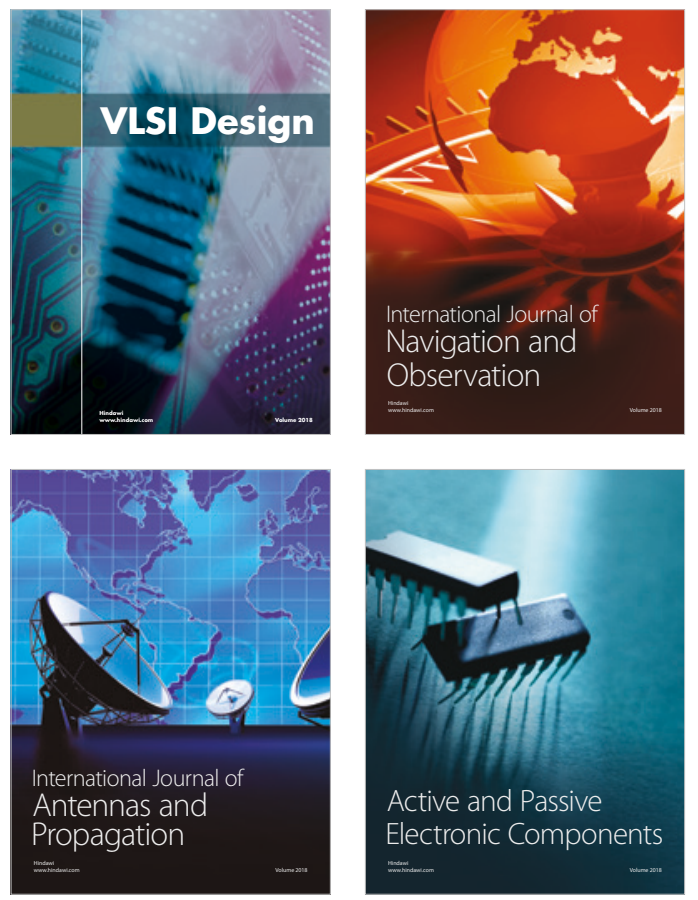
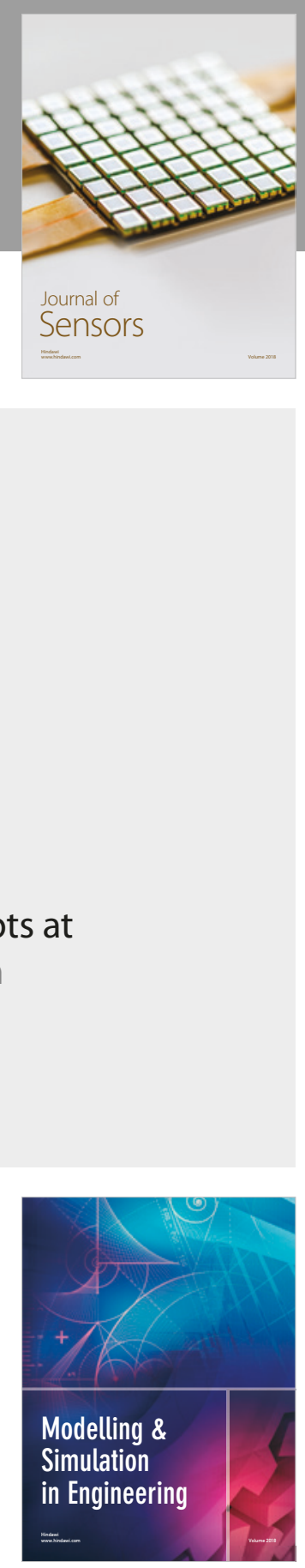

\section{Advances \\ Multimedia}
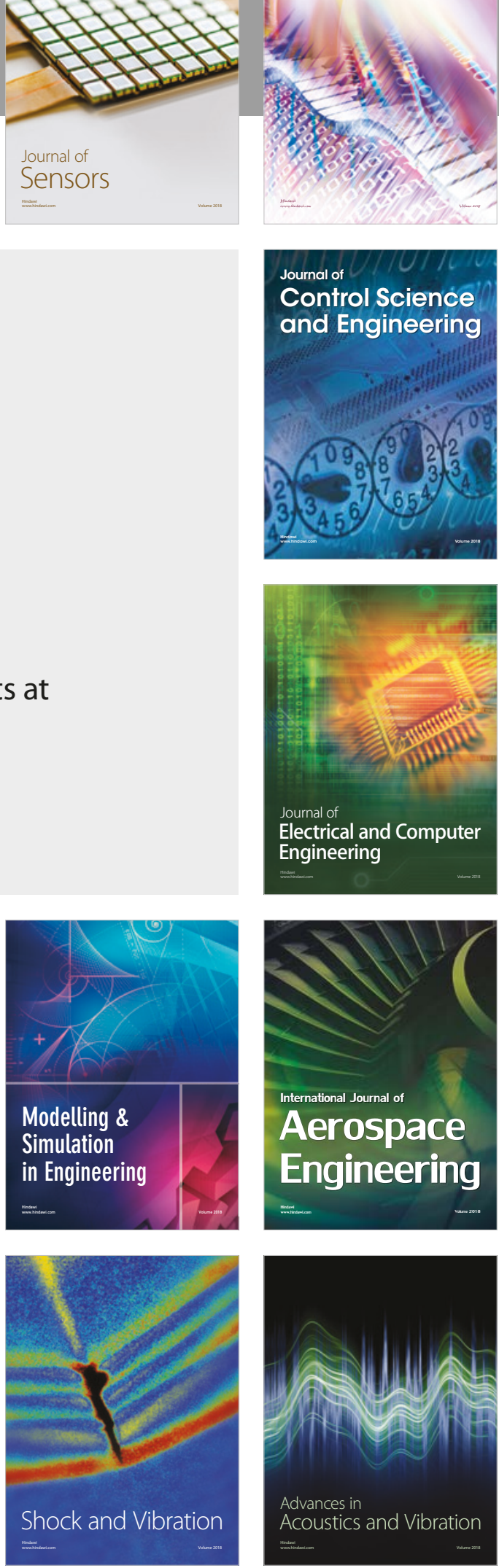